\title{
POUR UNE SOCIOLOGIE
}

\section{DES SITUATIONS RÉVOLUTIONNAIRES}

\author{
RETOUR SUR LES RÉVOLTES ARABES
}

\section{Mounia Bennani-Chraïbi et Olivier Fillieule}

\author{
«Les révoltes ont un corps avec lequel il est possible d'engager la lutte. \\ Les révolutions, en revanche, ont beaucoup en commun avec les spectres. » \\ Metternich à Guizot, 31 octobre 1847
}

La première image qui vient à l'esprit, lorsque l'on considère ce qui apparaît comme une cascade de soulèvements initiés par la chute rapide et inattendue de Ben Ali le 14 janvier 2011, c'est le «printemps des peuples » en 1848, lorsqu'une manifestation de rue à Paris débouche sur trois jours d'insurrection, la restauration de la République et, par la grâce du télégraphe, des chemins de fer et des presses rotatives, s'étend en une dizaine de jours à des villes aussi diverses que Berlin et Munich, Vienne, Milan, Venise.

Pourtant, comme pour 1848, il faut se méfier des interprétations trop globalisantes qui voudraient voir dans les événements de 2010-2012 le produit d'une vague unifiée d'insurrections. Au-delà de l'effet déclencheur de la chute de Ben Ali, les trajectoires et donc les formes prises par les soulèvements égyptien, marocain, bahreïni, libyen, yéménite et syrien sont très contrastées et ce n'est que par méconnaissance ou mu par un enthousiasme bien compréhensible que l'on a parlé de « révolutions arabes » ou de «arab uprisings ». Il est trop tôt pour savoir si l'on a à faire, ici ou là, à des révolutions. La question ne pourra être tranchée qu'a posteriori, à l'aune des résultats produits; elle ne nous occupera pas ici. Nous nous contenterons de parler de «révoltes», terminologie vague qui a l'avantage de ne préjuger en rien de ce que des processus en cours particulièrement complexes produiront. Pour autant, nous sommes conscients que des Tunisiens, des Égyptiens, des Libyens, des Yéménites, des Syriens pensent vivre une révolution, luttent pour la faire triompher, la continuer ou la protéger. Par ailleurs, bien que le qualificatif « arabe » charrie des implicites et des malentendus, nous nous sommes résolus à l'utiliser par simple commodité de langage.

La littérature consacrée aux révolutions en science politique, sociologie et histoire est particulièrement vaste. Et le rythme des publications à propos des événements récents est particulièrement soutenu. Pourquoi dès lors infliger au lecteur un dossier de plus sur le sujet ? Deux solides raisons justifient notre entreprise. D'une part, si les sciences sociales sont prolixes sur les causes et les conséquences des révolutions, elles le sont beaucoup moins sur les situations révolutionnaires elles-mêmes, leurs propriétés et les processus qui y conduisent. Les publications récentes, nous le montrerons dans cette introduction, n'y échappent pas. D'autre part, toutes les contributions de ce dossier reposent sur des enquêtes de terrain menées au cœur de la dynamique des événements qui s'attachent à comprendre comment les acteurs individuels et collectifs «pris » dans cette dynamique perçoivent les situations et orientent leurs conduites, ce qui là encore est plutôt rare dans la production existante.

Nous reviendrons dans cette introduction sur ce qui justifie ce double parti pris. Nous commencerons par une analyse critique de la littérature dont on nous pardonnera le caractère non exhaustif ${ }^{1}$. Nous expliciterons dans un second temps un certain nombre de propositions théoriques et de manières de faire en nous appuyant prioritairement sur les expériences empiriquement documentées. Au lecteur d'en mesurer la pertinence dans chacune des contributions de ce dossier.

\footnotetext{
${ }^{1}$ On trouvera cependant dans cette livraison une bibliographie thématique et un compte rendu critique des numéros de revue parus en français : voir infra, p. 926-939.
} 


\title{
MISÈRE DE LA SOCIOLOGIE DES RÉVOLUTIONS
}

\author{
«À se chercher de faux problèmes, on trouve la vraie misère. » \\ Daniel Desbiens, Maximes d'aujourd'hui
}

\section{Causalisme et ambition nomologique}

Les situations révolutionnaires frappent toujours les contemporains par leur nouveauté, la rapidité des bouleversements qu'elles entraînent et l'incertitude qu'elles instaurent pour le futur. Dans ces conditions, il n'est pas étonnant que rares soient ceux qui peuvent se flatter comme Marat d' «aborder la révolution avec des idées faites ${ }^{2}$ ou de prétendre avoir prédit l'effondrement de l'Empire soviétique ${ }^{3}$.

Pierre Favre a discuté avec précision la question de l'imprévisibilité dans l'ordre social ${ }^{4}$. Retenons-en ici trois conclusions : 1/ «s'agissant du futur, on se trouve face à un processus non achevé, en cours d'effectuation, et dont la connaissance ne peut, par nature, qu'être inachevée. La prévision n'est donc pas possible $»^{5} ; 2 /$ «il est des phases où un régime politique a une sensibilité structurelle extrême, et il s'effondre face à une manifestation révélatrice de son inconsistance ${ }^{6} ; 3 /$ l'imprévisibilité n'implique pas «que donc toute prévision est impossible et toujours démentie. La prévision, dont on ne saurait en pratique se passer, est seulement conditionnelle, ou si l'on veut probabiliste ${ }^{\prime}$.

Les chercheurs qui se sont penchés sur l'histoire et la sociologie des phénomènes révolutionnaires sont de plus en plus nombreux à partager ce point de vue, avec pour conséquence le rejet croissant de toute théorie générale des révolutions ${ }^{8}$. Il n'est pas inutile de rappeler à cet égard les deux symposiums organisés par la Société américaine de sociologie au début des années 1990 pour comprendre pourquoi les événements de 1989 surprirent autant . Si des voix s'y élèvent encore pour défendre certaines formes de prévisibilité, elles sont couvertes par une méfiance croissante que Charles Kurzman expose dans un livre au titre explicite ${ }^{10}$. Il y étend la critique à toute prétention à vouloir a posteriori reconstruire les schémas causaux permettant de déterminer les facteurs d'émergence des situations révolutionnaires. Rejetant tout causalisme, il propose une « anti-explication », qui en appelle à l'abandon de toute tentative de prédiction rétrodictive, au profit d'une attention aux expériences vécues des situations révolutionnaires. En d'autres termes, si l'on ne doute pas de ce que le réel advenu est par nature déterminé ", l'on reconnaît dans le même mouvement l'immense difficulté qu'il y a à l'élucider intégralement à partir d'une recherche de ses causes, le plus souvent lointaines ${ }^{12}$. Ces remarques pointent trois faiblesses de la littérature, celle de l'illusion étiologique ${ }^{13}$, celle de l'ambition nomologique et celle de l'absence d'une

¿Jean-Paul Marat, Les chaînes de l'esclavage, 1774,

<http://www.uqac.uquebec.ca/zone30/Classiques_des_sciences_sociales/index.html>.

${ }^{3}$ Randall Collins, «The Future Decline of the Russian Empire », dans Weberian Sociological Theory, New York, Cambridge University Press, 1986, p. 186-209.

Pierre Favre, Comprendre le monde pour le changer, Paris, Presses de Sciences Po, 2005.

'P. Favre, ibid., p. 61.

P. Favre, ibid., p. 171-173 ; voir aussi p. 195.

P. Favre, ibid., p. 198 (italiques dans le texte).

: Outre les nombreuses publications de Charles Tilly sur lesquelles nous reviendrons plus bas, citons à titre d'exemple Nikki Keddie, « Can Revolutions Be Predicted ? , dans Iran and the Muslim World, New York, New York University Press, 1995, p. 13-33 ; Jeff Goodwin, No Other Way Out, Cambridge, Cambridge University Press, 2001 ; François Chazel, «La place de la mobilisation dans une révolution inattendue. L'effondrement de l'Allemagne de l'Est », dans Du pouvoir à la contestation, Paris, LGDJ, 2003, p. 163-192.

- Les actes de la première réunion sont publiés dans Theory and Society, 23 (2), 1993. Ceux de la seconde dans American Journal of Sociology, 100 (6), mai 1995.

${ }^{10}$ Charles Kurzman, The Unthinkable Revolution in Iran, Harvard, Harvard University Press, $2^{\mathrm{e}}$ éd. 2005, p. 5-6 et p. 166.

"Pour parler comme Pierre Favre, le déterminisme méthodologique implique donc le contraire du causalisme : P. Favre, Comprendre le monde..., op . cit., p. 54 et p. 67-79.

12 Voir également Charles Tilly, "To Explain Political Processes », American Journal of Sociology, 100 (6), 1995, p. 1594-1610, dont p. 1601; Jean-Claude Passeron, Le raisonnement sociologique, Paris, Nathan, 1991, p. 367.

${ }^{13}$ Michel Dobry, Sociologie des crises politiques, Paris, Presses de Sciences Po, 1986. 
théorie de l'acteur ${ }^{14}$.

L'illusion étiologique renvoie à l'une des impasses majeures de la sociologie des révolutions is, laquelle, pour s'être focalisée sur la recherche du pourquoi de l'émergence des mobilisations, s'en tient le plus souvent à la recherche de déterminants lointains. Ceux-ci relevant soit des effets désorganisateurs du changement social, soit de l'ouverture de fenêtres d'opportunités. Cette focalisation se fait aux dépens d'une réflexion sur l'épaisseur des moyens mis en œuvre dans les interactions entre protagonistes et sur les «éventuelles médiations causales entre les "causes" suggérées et les caractéristiques de leurs supposés produits ${ }^{16}$. D'où la nécessité de bien distinguer avec Charles Tilly et Rod Aya les intentions, les résultats et les situations révolutionnaires ${ }^{17}$; celles-ci tendent à échapper aux conditions de leur genèse et leurs résultats ne permettent pas rétrospectivement d'en rendre raison ${ }^{18}$.

L'ambition nomologique - comment expliquer des séquences historiques dans la mesure où l'explication nécessite des lois liant les causes aux effets, lois dont l'histoire ne dispose pas - débouche sur une double forme de réductionnisme modélisateur. D'une part, les matrices explicatives sont souvent conçues à partir d'un exemple historique précis. Du coup l'extrapolation est de rigueur. Ainsi de Crane Brinton qui construit son modèle sur les phases de la Révolution française ${ }^{19}$ ou encore de Theda Skocpol qui retient 1789, 1917 et 1949 comme d'authentiques révolutions sociales portées par la classe paysanne ${ }^{20}$. D'autre part, parce qu'elles sont par définition à l'affût de schémas causaux récurrents, les théories générales réduisent la complexité des phénomènes au mieux à des stylisations et au pire à la recherche de causes uniques. C'est notamment le cas de Robert Palmer ou de Jacques Godechot ${ }^{21}$ ou encore, dans le champ des approches culturelles, des travaux de Patrice Higonnet qui survalorisent l'impact de la religion protestante et le rôle du « consensus anglosaxon ${ }^{22}$.

L'absence d'une théorie de l'acteur pointe quant à elle la difficulté de penser les liens entre micro et macro - comment expliquer un phénomène social alors que tout ce qui nous est donné à observer, ce sont des actions individuelles ? Trois dimensions explicatives dominent dans la littérature : les conditions structurelles (structure des opportunités politiques, densité des réseaux horizontaux et liens aux élites, griefs soudainement imposés, etc.), les idiomes culturels (cultural frames, Weltanschauung, traditions, etc.) et les structures de mobilisation (ressources matérielles organisationnelles et de leadership) ${ }^{23}$. Dans ce triptyque, les structures, la culture et la disposition de ressources expliquent les événements et, en retour, les événements peuvent induire des transformations des structures, de la culture et des ressources. Or, « dans ce cadre, la structure (assistée de la culture) contraint les actions des individus pour produire les événements et les événements poussent les individus à changer la structure. L'action individuelle est dans ce modèle le troisième homme entre structure et événement qui fait que l'action se déroule et que les choses changent $\gg{ }^{24}$.

\footnotetext{
${ }^{14}$ Rod Aya, «The Third Man ; or Agency in History ; or, Rationality in Revolution », History and Theory, 40, décembre 2001, p. 143-152.

${ }^{15}$ De fait, parmi les historiens, cette illusion est plus rare. Elle est clairement dénoncée par François Furet, Penser la Révolution française, Paris, Gallimard, 2009 (1" éd. : 1978), p. 40, 39 et 44.

${ }^{16}$ Michel Dobry, «Ce dont sont faites les logiques de situation », dans Pierre Favre, Olivier Fillieule, Fabien Jobard (dir.), L'atelier du politiste, Paris, La Découverte, 2007, p. 119-148, dont p. 131.

Charles Tilly définit la situation révolutionnaire, à partir du concept de «double pouvoir » chez Trotsky, comme toute situation de souveraineté multiple dans laquelle le pouvoir étatique fait l'objet de prétentions concurrentes soutenues par une fraction significative de la population et sans que le dit pouvoir puisse ou veuille réprimer la coalition oppositionnelle (Charles Tilly, From Mobilization to Revolution, Reading, AddisonWesley, 1978, p. 189-222).

${ }^{18}$ Rod Aya, Rethinking Revolutions and Collective Violence, Amsterdam, Het Spinhuis, 1990, p. 20.

${ }^{10}$ Crane Brinton, The Anatomy of Revolution, Londres, Jonathan Cape, 1953.

${ }^{20}$ Theda Skocpol, States and Social Revolutions. A Comparative Analysis of France, Russia, and China, New York, Cambridge University Press, 1979.

${ }^{21}$ Robert Roswell Palmer, The Age of the Democratic Revolution, Princeton, Princeton University Press, 1959 et 1964 ; Jacques Godechot, Les révolutions (1770-1799), Paris, PUF, 1963.

${ }_{22}$ Patrice Higonnet, Sisters Republics. The Origins of French and American Republicanism, Cambridge, Harvard University Press, 1988.

${ }_{23}$ Pour une illustration d'un tel triptyque explicatif, voir par exemple Theda Skocpol, Social Revolutions in the Modern World, Cambridge, Cambridge University Press, 1994.

${ }^{24} \mathrm{R}$. Aya, « The Third Man... », art. cité, p. 144.
} 
En réalité, peu de travaux échappent à l'illusion étiologique, au réductionnisme modélisateur et à l'absence d'une théorie explicite de l'acteur ${ }^{25}$ ce qui explique sans doute la très faible cumulativité des recherches, pourtant profuses, dans ce domaine.

\section{Où tout est dans tout et inversement}

Les travaux produits à chaud sur les «révoltes arabes » ne font pas exception. Le caractère imprévisible des événements sert de préambule quasi-rituel aux propositions de grilles explicatives ${ }^{26}$. Après s'être longtemps interrogé sur "les causalités d'un manque » démocratique ${ }^{27}$, il faudrait désormais se demander pourquoi les événements de 2011-2012 sonnent le glas de l' «exceptionnalité » ${ }^{28}$ du monde arabe et annoncent un nouveau seuil dans le processus d'universalisation du fait révolutionnaire ${ }^{2}$. À front renversé, ce sont bien souvent les mêmes «défauts » et les mêmes «anomalies " ${ }^{30}$ auparavant pointés comme autant de verrous à la démocratisation qui par la grâce du surgissement de l'histoire réelle deviennent les causes de la révolte et de ses succès.

Certes, l'ambition nomologique boude désormais les modèles monocausaux au profit d'approches dites «combinatoires » ${ }^{31}$. Mais celles-ci se contentent souvent d'agréger tout un ensemble de causes, facteurs, déterminants, censés rendre compte à différentes échelles de temps et d'observation, tant des intentions, des situations que des résultats révolutionnaires ${ }^{32}$.

Il n'est pas possible ici de rendre compte dans le détail de cette fièvre explicative. Pour s'en convaincre, le lecteur se reportera à la bibliographie thématique placée en fin de volume. Nous proposons plutôt ici un parcours libre et une mise en ordre à vertu pédagogique des principales grilles de lecture des révolutions: les lectures d'inspiration marxiste, les paradigmes structuralistes, les théories de la frustration relative et de la modernisation, les approches culturelles et diffusionnistes.

En tout premier lieu, les événements dans la région donnent un nouvel élan aux approches d'inspiration marxiste qui érigent la crise du capitalisme mondial en variable explicative centrale. L'autoritarisme des régimes du monde arabe ne serait que la «forme politique du capitalisme dans la région ${ }^{33}$, le «crony capitalism ${ }^{34}$ n'étant pas une « aberration » du système, mais une caractéristique des processus d'accumulation capitalistes dans le monde. De ce point de vue, le soulèvement égyptien marquerait l'aboutissement de trente ans de réformes néolibérales, imposées par des institutions financières internationales qui ont accentué la vulnérabilité du pays face à la crise mondiale, en provoquant la concentration des richesses entre les mains d'une toute petite minorité et en creusant le fossé entre les classes ${ }^{35}$. Cette lecture connaît des variantes. Pour les éditeurs d'un numéro spécial de Globalizations, les révoltes arabes constituent une facette de la «révolution globale »

\footnotetext{
${ }^{25}$ La place manque pour indiquer malgré tout un ensemble d'études de cas qui y échappent. Comme par exemple le livre de Wayne Te Brake (Shaping History. Ordinary People in European Politics, 1500-1700, Berkeley, University of California Press, 1998), remarquable par son attention au processus de politisation des groupes en révolution et aux constants décalages entre intentions initiales et actions dans les logiques de situation.

${ }^{26}$ Les coordinateurs du dossier publié par la revue Mouvements mettent en relation l'acuité de cet effet de surprise avec un orientalisme diffus percevant le monde arabe comme figé (Agnès Deboulet, Dimitri Nicolaïdis, «Les hirondelles font-elles le printemps ? », Mouvements, 66, 2011, p. 7-10).

${ }^{27}$ Ghassan Salamé, «Sur la causalité d'un manque : pourquoi le monde arabe n'est-il donc pas démocratique? », Revue française de science politique, 41 (3), juin 1991, p. 307-341.

${ }_{28}$ Sarah Ben Néfissa, «Mobilisations et révolutions dans les pays de la Méditerranée arabe à l'heure de "l'hybridation" du politique : Égypte, Liban, Maroc, Tunisie », Revue Tiers Monde, hors série, 2011, p. 5-24 .

${ }_{20}$ Par exemple, Hamit Bozarslan, «De quoi la révolution est-elle le nom ? Post-scriptum pour poursuivre la réflexion », dans Hamit Bozarslan, Gilles Bataillon, Christophe Jaffrelot, Passions révolutionnaires, Paris, Éditions de l'EHESS, 2011, p. 169-182.

${ }^{30}$ F. Gregory Gause III, "Why Middle East Studies Missed the Arab Spring ? The Myth of Autoritarian Stability », Foreign Afffairs, 90 (4), 2011, p. 81-90.

C. Kurzman, The Unthinkable Revolution in Iran, op. cit., p. 166.

${ }_{32}$ Par exemple, Jack Goldstone, «Toward a Fourth Generation of Revolutionary Theory », Annual Review of Political Science, 4, 2001, p. 139-187.

${ }_{33}$ Adam Hanieh, «Egypt's Uprising. Not Just a Question of Transition», The Bullet, 462, 2011, $<$ http://www.socialistproject.ca/bullet/462.php>.

${ }^{34}$ Formulation de Nader Fergany dans l'Arab Human Development Report (2002-2005).

${ }^{35}$ Lin Noueihed, Alex Warren, The Battle for the Arab Spring. Revolution, New Haven, Yale University Press, 2012, p. 112.
} 
contre le système économique libéral prédominant, contre les exclusions et les contradictions produites par le système-monde ${ }^{36}$. Elles seraient porteuses d'une aspiration à créer un monde alternatif "par le bas » ${ }^{37}$. Dans une perspective dépendantiste revisitée, les événements de 2010-2011 sont interprétés comme une nouvelle tentative de pays comme l'Égypte de se soulever contre leur «statut actuel de périphérie dominée » et contre les «exigences du libéralisme mondialisé ${ }^{38}$.

De telles approches ont le mérite de ne pas se focaliser sur l' «exceptionnalité » de la région et de proposer une lecture conflictualisée de la mondialisation. Néanmoins, comme cela a été souligné à maintes reprises, de tels paradigmes souffrent notamment de leur économicisme; tout découle mécaniquement de ce qui se joue sur le plan économique : les relations entre États, l'État lui-même, les classes sociales, les mouvements sociaux, etc.

Inversement, un autre ensemble de travaux subordonne la détermination économique au primat du politique. Dans une perspective structuraliste à la Skocpol ${ }^{39}$, l'autonomie de l'État est posée comme une condition de base, les déterminants de celui-ci, son effondrement ou sa crise sont mis au cœur de l'analyse. Au prolongement de telles approches, des auteurs tentent d'éclairer le contraste entre la chute des présidents tunisien et égyptien et la résilience des autres régimes de la région (avec un traitement particulier des cas libyen et syrien) : «vrais » et «faux » États-nation, monarchies «modérées» et inclusives par opposition aux républiques, sociétés fragmentées ou homogènes, rente pétrolière, histoires marquées par la violence, etc. ${ }^{40}$. Les révolutions viendraient débarrasser des régimes «trop fermés et trop ouverts, trop modernes et trop anachroniques, trop rationnels et trop "insensés" " ${ }^{41}$ des « ordures médiévales ». À partir de la centralité du conflit politique, des auteurs examinent les causes structurelles des «révoltes arabes» sous les deux facettes de la crise économique mondiale et du développement de la crise au sein de la sphère étatique.

Tout d'abord, à l'inverse des processus de démocratisation de la troisième vague, les « révoltes arabes » ne devraient rien à une impulsion démocratique occidentale. Bien au contraire, elles surviennent en dépit du traitement «exceptionnel » des régimes autoritaires arabes par les grandes puissances, obnubilées par le «spectre islamiste » ${ }^{42}$, soucieuses de préserver le statu quo dans le conflit israélo-palestinien et de maintenir leur influence sur la ressource pétrolière. Le ton est particulièrement acerbe ${ }^{43}$ et certains vont jusqu'à mettre en évidence les moyens mis en œuvre par les Occidentaux pour faire obstruction aux révolutions ${ }^{44}$. Les facteurs internationaux décisifs relèveraient plutôt de la sphère économique. L'augmentation mondiale des prix des aliments et de l'énergie aurait précipité le déclenchement des révoltes, la crise financière de 2007 étant «la goutte (ou l'océan) d'eau qui fit déborder la situation sociale ${ }^{45}$. L'extension de la crise dans la région tiendrait à la baisse des exportations, à la réduction des flux financiers vers les économies non pétrolières, à la chute du crédit, à l'amputation des programmes de développement nationaux et d'aide au développement ${ }^{46}$.

En second lieu, ces réformes, plutôt que de permettre le renouvellement des bases

\footnotetext{
${ }^{36}$ Anna M. Agathangelou, Nevzat Soguk, «Rocking the Kasbah. Insurrectional Politics, the "Arab Streets", and Global Revolution in the 21 Century », Globalizations, 8 (5), 2011, p. 551-558.

${ }^{37}$ Jackie Smith, «Globalizations Forum on Middle East Protests. Commentary », Globalizations, 8 (5), 2011, p. 655-659.

${ }_{38}$ Samir Amin, « 2011 : le printemps arabe ? », Mouvements, 67, 2011, p. 135-156, dont p. 139.

${ }^{39}$ D'après Theda Skocpol, trois éléments jouent un rôle primordial : le contexte international ; la crise fiscale et la division des élites ; les révoltes paysannes (T. Skocpol, States and Social Revolutions..., op. cit.).

${ }^{4}$ Par exemple, Georges Corm, «Première approche d'une contextualisation des révoltes populaires arabes », Confluences méditerranée, 79, 2011, p. 93-111.

" Hamit Bozarslan, «Réflexions sur les configurations révolutionnaires tunisienne et égyptienne », Mouvements, 66, 2011, p.11-21, dont p. 16.

${ }^{42}$ Luis Martinez, «Le printemps arabe, une surprise pour l'Europe », Projet, 322, 2011, p. 5-12.

${ }^{43}$ Voir la critique de l'ouvrage édité par les Foreign Affairs, The New Arab Revolt, New York, Council on Foreign Relations, 2011, par Raymond William Baker, «Understanding Egypt's Worldly Miracles », The Middle East Journal, 66 (1), 2012, p. 163-170, dont p. 165-167.

" Jeremy Salt, « Containing the "Arab Spring”", Interface, 4 (1), 2012, p. 54-66.

${ }^{45}$ Jean-François Daguzan, « De la crise économique à la révolution arabe », Maghreb-Machrek, 206, 2011, p. 915.

${ }^{46}$ Par exemple, El Mouhoub Mouhoud, «Economie politique des révolutions arabes : analyse et perspectives », Maghreb-Machrek, 210, 2011-2012, p. 35-47.
} 
sociales des régimes dans les pays non pétroliers, auraient élargi le rang des mécontents ${ }^{47}$; le retrait de l'État conduisant à la rupture des «pactes sociaux », jusque-là considérés comme l'une des bases de la résilience de l'autoritarisme ${ }^{48}$. L'accentuation de la corruption et de la prédation des économies nationales par des «cliques », issues de l'entourage des chefs d'État, auraient aussi exaspéré les élites marginalisées ainsi que l'armée dans le cas égyptien ${ }^{49}$. Sur le plan politique, la «délibéralisation ${ }^{50}$ se serait traduite par l'extension de la répression ${ }^{51}$ audelà des islamistes et par le faible accès de l'opposition à la sphère politique instituée. En Égypte, les amendements constitutionnels en vue des élections de novembre et décembre 2010, une étape clé dans la préparation de l'accession au pouvoir de Gamal Moubarak, ont suscité un large mouvement de boycott des élections, la place de l'opposition au sein du parlement reculant en deçà de son niveau de 2005. Les accès de la vie politique institutionnelle étant bloqués, l'occupation de la rue serait devenue la seule issue ${ }^{52}$.

Ces lectures ont pour intérêt de réintroduire l'État dans l'analyse, d'orienter le regard vers les relations entre les groupes sociaux, entre ces groupes et l'État, entre les États. Cependant, elles prêtent le flanc aux mêmes critiques que les approches structuralistes, à savoir la marginalisation des dimensions culturelles et idéologiques, la faible attention prêtée aux révoltes en elles-mêmes et plus globalement la négligence des processus.

Sur un autre plan, l'invocation des récentes vagues de mobilisation amène plusieurs auteurs à mobiliser les mécanismes de la frustration relative. Emblème du soulèvement tunisien, Mohamed Bouazizi est dans un premier temps présenté comme un «diplôméchômeur ». Une telle information (aussi fausse soit-elle) remobilise plus que jamais les lectures en termes de déclassement, de sentiment d'injustice et d'humiliation ${ }^{53}$. Les médias ont largement puisé dans ces lectures, graphiques et cartes interactives à l'appui ; The Economist Online a même publié « l'index du lanceur de chaussures ${ }^{54}$, construit sur la base d'un ensemble d'indicateurs statistiques.

Nous le soulignions il y a une dizaine d'années, les approches par la frustration «se déclinent sous plusieurs variantes: crise économique, "aliénation" en rapport avec les transformations accélérées connues par les sociétés du Maghreb et du Moyen-Orient qu'il s'agisse d'exode rural et d'urbanisation rapide ou de "rupture démographique" provoquée par l'arrivée massive sur les marchés de l'emploi, du logement, etc. des générations nées après les indépendances; échec de "l’État imité" ou de "l’État importé" [...]; amplification des sentiments d'exclusion par la mondialisation » ${ }^{55}$. Dans la même veine, dès les années 1980, la jeunesse des sociétés arabes est saisie «sous le prisme de la crise » et le chômage des diplômés est constitué en maillon central de la chaîne argumentative, plus ou moins longue, mobilisée pour expliquer une diversité de phénomènes : pression démographique ${ }^{56}$, difficulté d'insertion professionnelle et sentiment de déclassement, crise du logement, du mariage, économique, politique ${ }^{57}$.

${ }^{47}$ F. G. Gause III, «Why Middle East Studies Missed the Arab Spring ?...», art. cité, p. 86.

${ }^{48}$ Par exemple, Béatrice Hibou, «Économie politique et morale d'un mouvement social », Politique africaine, 121,2011, p. 5-22.

"Par exemple, Vincent Geisser, Abir Krefa, «L'uniforme ne fait plus le régime, les militaires arabes face aux "révolutions" ", Revue internationale et stratégique, 83, 2011, p. 93-102.

${ }^{50}$ Eberhard Kienle, A Grand Delusion. Democracy and Economic Reform in Egypt, Londres, Tauris, 2000.

"George Joffé, "The Arab Spring in North Africa. Origins and Prospects », The Journal of North African Studies, 16 (4), 2011, p. 507-532.

${ }^{52}$ L. Noueihed, A. Warren, The Battle..., op. cit., p. 106.

ss Pour une critique de ces lectures, voir entre autres Amin Allal, Youssef El-Chazli, « Figures du déclassement et passage au politique dans les situations révolutionnaires égyptienne et tunisienne », dans Ivan Sainsaulieu, Muriel Surdez (dir.), Sens politiques du travail, Paris, Armand Colin, 2012, p. 321-336.

${ }^{44}$ «Arab Unrest Index. The Shoe-Thrower's Index», The Economist Online, 9 février 2011, <http://www.economist.com/blogs/dailychart/2011/02/daily_chart_arab_unrest_index >.

${ }_{s s}$ Mounia Bennani-Chraïbi, Olivier Fillieule, «Appel d'air(e) », dans Mounia Bennani-Chraïbi, Olivier Fillieule (dir.), Résistances et protestations dans les sociétés musulmanes, Paris, Presses de Sciences Po, 2003, p. 17-42, dont p. 34.

${ }_{56}$ Emmanuel Todd, Allah n’y est pour rien! Sur les révolutions arabes et quelques autres, Loubiana, Arretsurimages.net, 2011 ; Emmanuel Todd, Youssef Courbage, Le rendez-vous des civilisations, Paris, Seuil, 2007.

"Mounia Bennani-Chraïbi, Iman Farag, «Constitution de la jeunesse dans les sociétés arabes: figures, catégories et analyseurs », dans Mounia Bennani-Chraïbi, Iman Farag (dir.), Jeunesses des sociétés arabes, 
De telles lectures pointent du doigt des phénomènes empiriquement observables et mesurables. Mais, elles soulèvent les mêmes objections que le paradigme de la frustration relative : caractère «volcanique » du modèle, prédominance d'une conception psychologique et peu sociologique, flou de la démonstration sur la nature des relations entre conditions objectives et perceptions déduites de manière tautologique, faiblesse de l'explicitation du passage de la privation relative à la mobilisation ${ }^{58}$, etc. Sans compter que cette notion fait traditionnellement recette dans les travaux sur les sociétés du Maghreb et du Moyen-Orient qu'il s'agisse d'expliquer les émeutes urbaines, les mouvements islamistes ou les attentatssuicides.

Actrice centrale des « révoltes arabes », la jeunesse ne s'incarne pas seulement dans la figure du diplômé chômeur mais aussi dans celle, plus glamour et mieux accordée au désir des commentateurs occidentaux, du jeune éduqué et moderne, féru de démocratie et de technologies numériques. Par le truchement de cette icône, le paradigme de la modernisation réapparaît en habits neufs ${ }^{59}$. Parce qu'elle se modernise, parce qu'elle s'ouvre à la culture démocratique occidentale via notamment ses produits culturels et l'accès à une information de plus en plus difficile à contrôler, la jeunesse «mondialisée » des sociétés arabes ne pouvait que porter un réveil démocratique inéluctable ${ }^{60}$. Le refrain n'est pas neuf. Si l'on excepte la parenthèse que marque la «fin du grand récit» développementaliste, lorsque la jeunesse devient « islamiste », « fanatisée », « terroriste », l'on n'est pas là si éloigné du lendemain des indépendances nationales, lorsque «la» jeunesse était pensée comme «le moteur du changement social et politique, et comme porteuse des valeurs de la modernité ${ }^{61}$. Seule nouveauté dans le discours tenu en 2011, l'apparition de nouveaux qualificatifs: cette jeunesse est dorénavant «connectée » et «mondialisée ». Grâce à sa créativité, la « rue arabe » s'érigerait désormais en modèle pour les indignés du monde ${ }^{6_{2}}$, montrant ainsi que « l'innovation politique peut surgir des périphéries ${ }^{63}$.

Dans ces approches, la technologie est constituée en agent actif des révolutions. Là encore, la thématique ne date pas d'aujourd'hui. Les théoriciens de la modernisation lui prêtaient déjà un rôle central dans la mise en œuvre du changement social. Mais, avec le développement des nouvelles technologies de l'information et de la communication (NTIC), le rythme des publications sur le sujet n'a fait que croître, périodiquement réactivé par des événements comme l'insurrection zapatiste en 1994, le mouvement altermondialiste, la coalition contre la guerre en Irak en 2003 et bien entendu les révoltes iraniennes ou moldaves de 2009, hâtivement qualifiées de «twitter Revolutions » ${ }^{64}$. Avec la surmédiatisation du rôle joué par les appels à la mobilisation via Facebook en 2011, les expressions « Facebook Revolution », «Révolution $2.0 »$ ou « Wikileaks Revolution» (pour le cas tunisien) se sont imposées. Pour les «cyberphiles» ou les «cyberutopiques», les nouvelles technologies de l'information et de la communication démocratiseraient la production de l'information et favoriseraient une diffusion quasi instantanée de textes, d'images, de vidéos au sein des

Paris, Aux lieux d'être, 2007, p. 11-47, dont p. 18 et suiv.

58 Philippe Corcuff., « Frustrations relatives », et Isabelle Sommier, « Privation relative », dans Olivier Fillieule, Lilian Mathieu, Cécile Péchu (dir.), Dictionnaire des mouvements sociaux, Paris, Presses de Sciences Po, 2009, p. 242-248, et p. 441-448.

s9 Pour Samuel Huntington, les révolutions sont une composante de la modernisation. Elles répondent à un processus de modernisation trop rapide et mal maîtrisé, lorsqu'il y a désajustement entre des secteurs de la société qui se modernisent à des vitesses différentes et, plus précisément, lorsque le développement des institutions politiques est en retard par rapport au changement social et économique (Samuel Huntington, Political Order in Changing Societies, New Haven, Yale University Press, 1968).

"o Voir à titre d'exemple l'enthousiasme affiché pour «Les jeunes Egyptiens connectés, éduqués, audacieux [qui] guident la révolte » («Wired, Educated an Shrewd, Young Egyptians Guide Revolt ») titre du New York Times, du 10 février 2011, p. 1. Voir également Farhad Khosrokhavar, The New Arab Revolutions that Shook the World, Boulder, Paradigm Publishers, 2012.

${ }^{6}$ F. Khosrokhavar, ibid., p. 17.

${ }^{62}$ Georges Corm, «Quand la rue arabe sert de modèle au Nord », Le Monde, 11 février 2011.

${ }^{63}$ Yves Gonzalez-Quijano, «Les "origines culturelles numériques" des révolutions arabes », 23 mars 2011, p. 4, $<$ http://www.ceri-sciences-po.org/archive/2011/mars/dossier/art_ygq.pdf>.

${ }^{6}$ On se reportera à la bibliographie thématique en fin de livraison pour des références. Pour une approche synthétique des liens entre médias et protestation, voir Érik Neveu, « Médias, mouvements sociaux, espaces publics », Réseaux, 17 (98), 1999, p. 17-86; et plus particulièrement sur les NTIC : Dominique Cardon, Fabien Granjon, Médiactivistes, Paris, Presses de Sciences Po, 2010. 
espaces nationaux et au-delà, contournant ainsi le monopole des médias conventionnels. De même, elles permettraient de résoudre la plupart des problèmes liés à la mobilisation en contexte répressif : "Cette technique nouvelle représente sinon la mort, du moins l'agonie de Que faire? de Lénine. La fonction d'organisation disparait à mesure qu'un clic permet de mettre en relation des individus et d'orchestrer une mobilisation ${ }^{65}$. Quant aux critiques du « cyberutopisme », elles minimisent le rôle joué par les NTIC dans le déclenchement et l'organisation des révoltes, démystifient le pouvoir libérateur d'Internet ${ }^{6}$ et rappellent que le contre-espace public virtuel peut être "une autre ruse du régime orwellien » ${ }^{67}$ et que les technologies ne font pas les révolutions ${ }^{68}$.

Cette double focalisation du discours médiatique et scientifique sur la jeunesse «mondialisée » et sur la «révolution 2.0 » réactive la dénonciation d'une conception du changement unilinéaire, téléologique. Elle relance par ailleurs le débat sur le néoorientalisme. Ainsi, pour Rabab El-Mahdi, le fait même de construire la révolte comme « jeune », «non violente » et d'ériger les médias sociaux comme « champions » repose sur une lecture orientaliste « binaire » visant à confirmer la suprématie de l'Occident, en opposant «la » tradition - associée à l'obscurantisme, à la violence et à tout ce qui fait obstacle au progrès - et « la » modernité « importée », libératrice ${ }^{9}$.

L'épistémé de la modernité se retrouve partiellement dans les lectures préoccupées des facteurs culturels « qui rendent pensable et souhaitée une si radicale rupture ${ }^{70}$, ou portant sur les changements dans les formes de l'obéissance et sur les cultures de résistance qui favorisent la révolte. Dans une comparaison des «origines culturelles » des révolutions française et anglaise, Roger Chartier discute cinq grands facteurs identifiés par Lawrence Stone $^{71}$ : la transformation du rapport au religieux ; l'importance de la référence juridique ; un « idéal culturel » marqué par la disqualification de la cour et de la capitale; un état d'esprit marqué par l'érosion de l'autorité de la famille, de l'État et de l'Eglise; une frustration intellectuelle en lien avec un «développement excessif de l'instruction ». La littérature récente ne manque pas de recourir implicitement à cette grille de lecture.

Les manières de nommer trahissent d'entrée la centralité accordée aux valeurs et aux idées : révolutions « modernes », « démocratiques », « civiles », «postmodernes » ${ }^{22}$, «postislamistes ${ }^{73}$, etc. En rupture avec les générations politiques précédentes (nationalistes et islamistes), les protestataires seraient porteurs d'une révolution culturelle et intellectuelle ${ }^{74}$. Ces femmes et ces hommes nouveaux auraient tissé une «nouvelle relation à soi, à autrui, au monde, et en particulier à l'Ouest ${ }^{75}$, à l'autorité politique, religieuse et familiale ${ }^{76}$. L'heure est au triomphe de l'individualité sur l'ordre communautariste confessionnel, sectaire ou tribal. Cette "démocratisation des esprits " " puiserait ses racines dans la constitution d'une

\footnotetext{
${ }_{65}$ Bertrand Badie, « Printemps arabe : un commencement », Études, 415 (7-8), 2011, p. 7-18, dont p. 15.

${ }^{6}$ Evgeny Morozov, The Net Delusion. The Dark Side of Internet Freedom, New York, Public Affairs, 2010.

${ }^{6}$ Roshanak Shaery-Eisenlohr «From Subjects to Citizens ? Civil Society and the Internet in Syria », Middle East Critique, 20 (2), 2011, p. 127-138, dont p. 137. Sur les arguments présentés par les «cyberphiles »et les « cyberskeptics », voir, par exemple, James L. Gelvin, The Arab Uprisings. What Everyone Needs to Know, Oxford, Oxford University Press, 2012, p. 50 et suiv.

"Par exemple, Sahar Khamis, Paul B. Gold, Katherine Vaughn, «Beyond Egypt's "Facebook Revolution" and Syria's "YouTube Uprising" ", Arab Media \& Society, 15, 2012, $<$ http://www .arabmediasociety.com/?article=791>.

${ }^{6}$ Rabab El-Mahdi, "Orientalising the Egyptian Uprising », Jadaliyya, avril 2011, <http://www.jadaliyya.com/pages/index/1214/orientalising-the-egyptian-uprising>. Voir aussi Greg Burris, "Lawrence of E-rabia. Facebook and the New Arab Revolt», Jadaliyya, octobre 2011, <http://www.jadaliyya.com/pages/index/2884/lawrence-of-e-rabia_facebook-and-the-new-arab-revo>.

${ }^{70}$ Roger Chartier, Les origines culturelles de la Révolution française, Paris, Seuil, 1990, p. 247.

"Lawrence Stone, The Causes of the English Revolution, 1529-1642, Londres, Ark Paperbacks, 1972.

${ }^{2} \mathrm{~S}$. Ben Néfissa, «Mobilisations et révolutions... », art. cité.

"Olivier Roy, «Des révolutions post-islamistes », Le Monde, 12 février 2011.

${ }^{74}$ Jeffrey C. Alexander, Performative Revolution in Egypt. An Essay in Cultural Power, Londres, Bloomsbury Academic, 2011, p. 18.

${ }^{75}$ F. Khosrokhavar, The New Arab Revolutions..., op. cit., p. 10.

${ }^{76}$ Y. Gonzalez-Quijano, «Les origines culturelles... », art. cité, p. 10. Sur la démocratisation du religieux, voir Dale F. Eickelman, James P. Piscatori, Muslim Politics, Princeton, Princeton University Press, 1996.

Mohamed Nanabhay, Roxane Farmanfarmaian, «From Spectacle to Spectacular. How Physical Space, Social Media and Mainstream Broadcast Amplified the Public Sphere in Egypt's "Revolution" », The Journal of North African Studies, 16 (4), 2011, p. 573-603.
} 
sphère publique arabe, notamment par la grâce d'Al Jazeera, laquelle aurait contribué à façonner " un nouveau public arabe ${ }^{78}$, à contourner les médias aux ordres, à promouvoir un pluralisme éditorial inédit en laissant place à «l'opinion... et l'autre opinion » (ar-ra'y wa arra'y al-akhar) ${ }^{79}$. Dans le même esprit, des travaux soulignent le rôle joué par les discours des coulisses ${ }^{80}$ (rumeurs, anecdotes) et par un ensemble d'objets culturels - des clips, des films, des séries télévisées, des essais, des romans ${ }^{81}$, des pièces de théâtre, des blogs, etc. - dans la diffusion de valeurs libérales et dans la délégitimation des pouvoirs en place à travers la critique de l'autoritarisme et de la corruption ; «la disgrâce du chef ${ }^{82}$ précéderait ainsi la chute de celui-ci.

Ces approches mettent en évidence l'impact des idées et des valeurs, si marginalisées par un ensemble de paradigmes. Reste cependant à « mettre au jour leur dynamique et [à] ne pas négliger leur rôle au niveau et au sein même de l'action, c'est-à-dire leur "efficace" [...] une "logique de discours" n'est encore pas "une logique d'action" $\gg{ }^{83}$.

Nous terminerons ce rapide tour d'horizon des publications consacrées aux « révoltes arabes » par un leitmotiv en forme d'hommage à Dwight Eisenhower. La chute de Hosni Moubarak quatre semaines après celle de Zine El-Abidine Ben Ali a déclenché une épidémie d'expressions imagées comme «effet domino», «contagion», «tsunami », «vague déferlante », «boule de neige », etc. Quelles que soient par ailleurs les chaînes causales retenues, elles incluent presque toujours la question de la diffusion. Aux débats habituels sur cette question s'ajoute ici une spécificité. L'impact fédérateur des causes «arabes » ou « islamiques » a souvent conduit les analystes à postuler l'existence d' " une "rue arabe ou musulmane", une "opinion arabe ou musulmane" prête à se mobiliser et à se lever comme un seul homme, de manière uniforme, avec une définition des enjeux, des motivations, et des modes d'action identiques ${ }^{84}$. Tout en dénonçant l'homogénéisation factice que charrie l'usage de l'attribut «arabe» pour qualifier les « révoltes», les chercheurs conviennent qu'une «communauté imaginée »s5 arabe s'est façonnée. Elle aurait perduré en se recomposant grâce aux échanges favorisés par une communauté de langue, de civilisation, d'expériences historiques, et intensifiés par le pèlerinage, les voyages, les migrations de travail et les médias. De ce point de vue, la chaîne satellitaire Al Jazeera serait le véhicule d'un nouveau panarabisme porté par les individus ${ }^{86}$, là où «La Voix des Arabes », station de radio créée par Gamal Abdel Nasser en 1953, aurait été l'emblème d'un nationalisme arabe impulsé par les États.

Si, jusqu'à présent, la transnationalisation des mobilisations dans la région a surtout été appréhendée au prisme des mouvements islamistes et d'Al Qaïda ${ }^{87}$, aujourd'hui c'est plutôt sur le rôle des réseaux transnationaux ${ }^{88}$, des fondations et des organisations occidentales dans la diffusion des idées démocratiques que l'on met l'accent. De là à ériger l'universitaire américain et activiste Gene Sharp en inspirateur des révolutions arabes, il n'y a qu'un pas que beaucoup s'empressent de franchir ${ }^{89}$ : des Égyptiens et des Tunisiens auraient été initiés à la

\footnotetext{
${ }^{78}$ Miles Hugh, Al Jazeera, Londres, Abacus, 2005.

79 Claire-Gabrielle Talon, «Al Jazeera, un objet médiatique original. Une critique des normes journalistiques occidentales », Les Temps modernes, 664, 2011, p. 55-62, dont p. 59. Voir aussi Yves Gonzalez-Quijano, Tourya Guaaybess, Les Arabes parlent aux Arabes, Paris, Sindbad/Actes Sud, 2009 ; Marc Lynch, Voices of the New Arab Public, New York, Cambridge University Press, 2006.

${ }^{s o}$ James C. Scott, Domination and the Arts of Resistance, New Haven, Yale University Press, 1990.

${ }^{8}$ Alaa El Aswany, L'immeuble Yacoubian, Paris, Actes Sud, 2006.

${ }^{2}$ Voir à ce sujet l'article séminal de Michel Camau, «La disgrâce du chef. Mobilisations populaires arabes et crise du leadership », Mouvements, 66, 2011, p. 22-29.

${ }^{83}$ François Chazel, «Les ruptures révolutionnaires. Problèmes de définition », dans Madeleine Grawitz, Jean Leca (dir.), Traité de science politique, Paris, PUF, vol. 2, 1985, p. 635-646, dont p. 652.

${ }^{*}$ Mounia Bennani-Chraïbi, «Les conflits du Moyen-Orient au miroir des communautés imaginées : la rue arabe existe-t-elle ? Cas du Maroc », A Contrario, 5 (2), 2008, p. 147-156.

${ }^{8}$ Benedict Anderson, Imagined Communities. Reflections on the Origin and Spread of Nationalism, Londres, Verso, 1991.

${ }_{86}$ Par exemple, Bichara Khader, «Le “printemps arabe” : un premier bilan », Alternatives Sud, 19, 2012, p. 7-39.

${ }^{87}$ Par exemple, Olivier Roy, Globalized Islam. The Search for a New Ummah, New York, Cambridge University Press, 2004.

${ }^{88}$ Par exemple, Maha Abdelrahman, «The Transnational and the Local : Egyptian Activists and Transnational Protest Networks », British Journal of Middle Eastern Studies, 38, 2011, p. 407-424.

${ }^{\circ}$ Par exemple, David D. Kirkpatrick, David E. Sanger, « A Tunisian-Egyptian Link That Shook Arab History »,
} 
lutte pacifiste grâce à son célèbre guide pratique, From Dictatorship to Democracy, et aux formations qu'ils ont suivies dans le Centre pour l'action et les stratégies non violentes appliquées (CANVAS), créé à Belgrade par le mouvement Otpor, ou dans l'Académie du Changement au Qatar ${ }^{\circ}$.

Il ne s'agit nullement de rejeter toute lecture diffusionniste d'un revers de main. Bien au contraire, nous invitons à relancer le débat avec les chercheurs qui ont renoncé aussi bien au causalisme qu'aux focales macrosociologiques. En effet, il serait fructueux d'articuler l'étude des processus cognitifs avec les analyses des réseaux et les approches séquentielles des carrières militantes, afin de mieux restituer les «processus vécus de diffusion », les modalités de relocalisation des idées, des causes et des performances ${ }^{9}$.

Que dire de cette revue partielle et trop rapide de la littérature ? Se féliciter d'abord de la réactivité de la communauté scientifique face à des événements majeurs qui viennent bouleverser bien des théories et bien des thèses désormais fragiles. Souligner le caractère stimulant de quelques travaux cités. Pourtant, force est de constater qu'à peu près aucune des impasses pointées plus haut n'a été véritablement résolue. La majorité des travaux reste enfermée dans la prédiction rétrodictive et la poursuite de schémas causaux macrosociologiques. Plusieurs causes possibles sont invoquées et, souvent, « tout est dans tout», sans que la prééminence d'un ordre de causalité soit véritablement démontrée. Resterait à établir si cela tient au poids des traditions de recherche respectives, ou plus généralement, pour reprendre une expression de Pierre Favre, à l'évaporation de la preuve en sociologie. Sur un autre plan, l'analyse des situations révolutionnaires, dans le sens d'une élucidation des processus internes à ces situations et des modes de leur enchaînement, demeure largement inexplorée ; les acteurs individuels sont partout présents, mais en ombre chinoise. Dès lors, que faire ? Très modestement, éliminer la recherche de causes, focaliser l'attention sur les situations, sur ce que les individus font dans ces situations et tâcher de retracer les processus typiques qui y conduisent.

\section{PERFORMANCES ET PROCESSUS}

«It is the details that theories in history have to grasp if they are to be any good. » ${ }^{92}$

Ce dossier porte sur l'analyse des situations révolutionnaires, entendues comme situations de double pouvoir ${ }^{93}$, aux fins d'identifier les séquences d'action - définies ici comme des performances, soit un ensemble agrégé d'interactions entre tous les acteurs partie prenante d'un échange de coup - qui y conduisent. La notion de performance, également d'inspiration tillyienne ${ }^{94}$, indique que les acteurs s'appuient sur des formes apprises, mais les interprètent, éventuellement les subvertissent ou s'en détachent, en fonction de logiques dont les déterminants sont à trouver dans l'échange de coups. Nous faisons ensuite l'hypothèse que l'observation de l'échange de coups doit permettre d'identifier des processus, que nous définissons comme des enchaînements susceptibles d'altérer les relations entre des ensembles donnés d'éléments, de manière identique ou proche, dans une variété de contextes. Ces processus peuvent être relationnels (au niveau meso des relations entre individus et groupes) ou cognitifs (au niveau micro des perceptions individuelles) ${ }^{95}$. Étudier les performances

\footnotetext{
The New York Times, 13 février 2011.

${ }^{\circ}$ Les principaux concernés n'ont pas tardé à mener une contre-offensive humoristique sur Twitter, sous le titre de «GeneSharpTaughtMe» [Gene Sharp m'a appris]. Voir Ould Mohamedou Mohammad-Mahmoud, «La démocratie arabe au regard du néo-orientalisme », Revue internationale et stratégique, 83, 2011, p. 85-91, dont p. 86.

"Isabelle Sommier, «Diffusion et circulation des mouvements sociaux», dans Olivier Fillieule, Éric Agrikoliansky, Isabelle Sommier (dir.), Penser les mouvements sociaux. Conflits sociaux et contestation dans les sociétés contemporaines, Paris, La Découverte, 2010, p. 101-120, dont p. 115.

92 Arthur L. Stinchcombe, Theoretical Methods in Social History, New York, Academic Press, 1978, p. 124.

${ }^{93}$ Soit la définition donnée en note 17 par Charles Tilly.

${ }^{94}$ Charles Tilly, Contentious Performances, Cambridge, Cambridge University Press, 2008.

${ }_{95}$ Nous reprenons ici une distinction introduite par Arthur Stinchcombe dans sa discussion de la notion de mécanisme, dans The Logic of Social Research, Chicago, Chicago University Press, 2005. Celui-ci ajoute le niveau des mécanismes environnementaux, lequel se réfère selon nous à des processus de long terme et répondant donc à des régimes différents de plasticité (pour employer une métaphore géologique). Ainsi, des
} 
revient donc à retenir les faits les plus saillants de certaines séquences ou des différences significatives entre des séquences et à les expliquer en identifiant dans ces séquences des processus robustes de portée relativement générale. Plus précisément, au-delà de l'établissement des conditions de départ, ou si l'on préfère du contexte, il faut s'attacher à identifier et décrire les processus conduisant ou pas à une situation révolutionnaire laquelle ne saurait découler directement des dites conditions de départ.

Un tel découpage est largement en affinité avec l'ambitieux projet qui anime Doug McAdam, Sidney Tarrow et Charles Tilly dans leur tentative de refonder le modèle du processus politique autour de la recherche de mécanismes propres à tels ou tels types d'épisodes ${ }^{9}$. Si nous partageons leur souci d'une approche dynamique, il nous semble toutefois qu'au-delà de ses ambiguïtés ${ }^{97}$, leur modèle demeure ouvertement causaliste et encore trop largement structuraliste.

Notre perspective repose par ailleurs sur trois postulats qui nous éloignent d'autant des attendus de Dynamics of Contention. D'une part, nous pensons que c'est en partant de ce que sont, font et disent les acteurs en situation que l'on peut spécifier de quoi sont faites les situations révolutionnaires et donc définir et observer des processus. Pour nous, les faits macrosociaux ne sont rien d'autre que les conséquences émergentes des actions individuelles, dans une logique au fond weberienne ${ }^{98}$.

D'autre part, contre une tendance lourde de la sociologie des mouvements sociaux à se cantonner aux seuls mouvements et acteurs contestataires et à leur relation à l'État ${ }^{99}$, nous estimons que les processus et les performances se construisent de manière relationnelle et appellent une prise en compte de tous les acteurs en présence dans un espace de conflit donné ${ }^{100}$.

Enfin, la révocation de la notion de cause nous conduit à considérer, avec Pierre Favre, que « le travail de la science consiste à considérer deux états successifs d'un système, à en isoler certaines caractéristiques pertinentes et à dégager des règles de transformations entre

mécanismes environnementaux comme la nationalisation des champs politiques ou encore la dédifférenciation s'inscrivent dans le temps long et, à l'échelle de l'étude des situations révolutionnaires, doivent plutôt être pensés comme des éléments conductifs (pour écrire comme Smelser) présents en dehors du cadre temporel de la crise. Le terme ainsi précisé inclut donc aussi bien des processus individuels (par exemple, les jeux d'assurance) et des mécanismes méso sociologiques. Nous le montrerons plus bas.

${ }_{9}^{\circ}$ Doug McAdam, Charles Tilly, Sidney Tarrow, Dynamics of Contention, Cambridge, Cambridge University Press, 2001; et aussi « Methods for Measuring Mechanisms of Contention », Qualitative sociology, 31, 2008, p. 307-331. Notre définition de la notion de processus reprend d'ailleurs la définition tillyienne du mécanisme (Charles Tilly, «L'analyse historique des processus politiques », dans Pascale Laborier, Danny Trom (dir.), Historicités de l'action publique, Paris, PUF, 2003, p. 23-57, dont p. 27.

${ }^{97}$ Notamment, la distinction entre les notions d'épisode, de processus et de mécanisme n'est pas consistante, comme plusieurs commentateurs l'ont souligné. Voir notamment les critiques rassemblées dans un dossier de Mobilization, 8, 2003 et dans le symposium sur «Measuring Mechanisms of Contention » dans Qualitative Sociology, 31, 2008.

${ }^{\circledR}$ R. Aya, «The Third Man... », art cité ; William H. Sewell Jr., « Three Temporalities : Toward an Eventfull Sociology », dans Terrence J. McDonald (dir.), The Historic Turn in the Human Sciences, Ann Arbor, Michigan University Press, 1996, p. 245-280. Dans «Methods for Measuring Mechanisms of Contention » (art. cité.), D. McAdam, S. Tarrow et Ch. Tilly dressent une liste des moyens d'identifier les mécanismes (pour eux) ou les processus (pour nous) qui, certes, insiste sur l'observation ethnographique des échanges de coups mais inclut également la «protest events analysis » et les méthodes statistiques de mesure indirecte, au risque encore une fois d'une certaine inconsistance théorique.

${ }^{99}$ Voir Verta Taylor, « Plus ça change, plus c'est la même chose », Mobilization, 8, 2003, p. 122-126, et Olivier Fillieule, «De l'objet de la définition à la définition de l'objet. De quoi traite finalement la sociologie des mouvements sociaux ?», Politique et Sociétés, 28 (1), 2009, p. 15-36 pour des critiques développées de cet aspect de la « contentious politics ».

${ }_{100}$ La prise en compte équilibrée de l'échange de coup entre les différents protagonistes est au cœur d'un ensemble de travaux qui, à partir de recherches sur les logiques du maintien de l'ordre (Olivier Fillieule, Donatella Della Porta (dir.), Police et manifestants, Paris, Presses de Sciences Po, 2006), s'étendent aujourd'hui aux autres arènes du monde social. Voir à ce propos l'ambitieux programme de recherche énoncé par James Jasper dans «A Strategic Approach to Collective Action. Looking for Agency in Social-Movement Choices », Mobilization, 9 (1), 2004, p. 1-16, formalisé dans Getting your Way. Strategic Dilemnas in the Real World, Chicago, The University of Chicago Press, 2006, et mis en œuvre dans Jan W. Duyvendak, James M. Jasper (eds), Players and Arenas. The Interactive Dynamics of Protest, à paraître. Voir également, dans une autre perspective, Neil Fligstein, Doug McAdam, A Theory of Fields, New York, Oxford University Press, 2012. 
ces deux états ${ }^{101}$. Chaque séquence d'action est travaillée par des processus qui conduisent à des résultats (de nouveaux équilibres), lesquels entrent à nouveau dans la définition des éléments environnementaux, relationnels et cognitifs nourrissant les calculs dans la séquence qui la suit. Dans cette conception cumulative qui se rapproche par certains aspects de la notion d'intrigue chez Paul Veyne ${ }^{102}$, les choix précédents contraignent les choix subséquents comme au jeu d'échec ${ }^{103}$.

Dans ce qui suit, un bref rappel de quelques propriétés des situations révolutionnaires nous amènera à préciser la question de la place qu'y occupe l'agency, pour enfin nous arrêter à la dimension sur laquelle les contributions à ce dossier insistent le plus, celle des performances. Ce parcours nous permettra de conclure sur l'existence d'un certain nombre de processus, donc de règles de changements d'états, propres à conduire aux situations révolutionnaires.

\section{Extension des mobilisations et logiques des coalitions}

L'extension de la contestation du pouvoir à une fraction significative de la population, et donc le brouillage des frontières habituellement plus ou moins étanches entre espaces sociaux, conditionne la situation révolutionnaire. La plupart des études de cas y identifient un petit nombre de propriétés récurrentes : la division ou la défection au sein des organes centraux de l'État; la division ou la défection au sein des forces de sécurité ; la constitution de larges coalitions transclassistes débouchant sur l'extension des mobilisations; la modularité enfin de la mise en forme des luttes (c'est-à-dire les cadrages de la situation comme les répertoires tactiques).

M. Dobry a proposé de formaliser plusieurs de ces propriétés, autour des notions de mobilisation multisectorielle et de fluidité politique ${ }^{104}$. Les situations de crise politique y sont comprises comme l'extension d'une mobilisation à différents secteurs de la société, au point d'en perturber sévèrement la marche routinière. S'ouvre alors un moment de fluidité politique dans lequel ces secteurs tendent à ne plus fonctionner selon leur logique ordinaire: ils deviennent beaucoup plus dépendants les uns des autres et l'incertitude prévaut, dans la mesure où les règles du jeu habituelles dans chaque secteur ne sont plus respectées.

Décrire les propriétés des situations révolutionnaires ne suffit pas. Encore faut-il s'attacher à comprendre les contextes qui les favorisent et les processus qui y conduisent. Ce que fait Misagh Parsa lorsqu'il estime qu'il faut d'abord faire porter l'analyse sur ce qui permet la constitution de coalitions transclassistes ${ }^{105}$. À partir d'une étude des stratégies déployées par les étudiants, le clergé, les ouvriers, et les capitalistes dans les révolutions en Iran, au Nicaragua, et aux Philippines, il identifie d'une part des facteurs facilitateurs structurels liés à la forme de l'État (plus ou moins interventionniste et plus ou moins exclusif) et à la forme prise par le conflit de classe (plus ou moins polarisé); d'autre part des facteurs renvoyant à la dynamique même de l'action, puisque le conflit une fois déclenché, les logiques de la situation échappent en partie aux déterminants qui l'ont produite. Par exemple, le recours à la répression, selon les formes qu'elle prend, peut accélérer ou freiner la formation de coalitions larges et donc l'extension de la mobilisation ${ }^{106}$.

\footnotetext{
${ }^{101}$ P. Favre, Comprendre le monde..., op. cit., p. 72.

${ }^{102}$ Paul Veyne, Comment on écrit l'histoire, Paris, Points Seuil, 1971, p. 51-53. Sur la causalité cumulative, voir Arthur L. Stinchcombe, Theoretical Methods in Social history, New York, Academic Press, 1978, p. 61-63, et R. Aya, «The Third Man...», art. cité, p. 147-148.

103 Pour une mise en œuvre de cette conception de l'analyse multiniveau et de la causalité inspirée de l'interactionnisme symbolique, voir Olivier Fillieule, «Disengagement from Radical Organizations. A Process and Multi-Level Model of Analysis », dans Bert Klandermans, Cornelius Van Stralen (eds), Movements in Times of Transition, Minnesota, Minnesota University Press, à paraître.

${ }^{104} \mathrm{M}$. Dobry, Sociologie des crises politiques, op. cit.

${ }^{105}$ Misagh Parsa, States, Ideologies, \& Social Revolutions. A Comparative Analysis of Iran,. Nicaragua, and the Philippines, Cambridge, Cambridge University Press, 2000 (voir le compte rendu de l'ouvrage infra, p. ?- ?). Charles Tilly ne dit pas autre chose lorsqu'il analyse les alliances entre différentes fractions de classe dans les révolutions de 1830 et 1848 en France (From Mobilization..., op. cit.).

${ }^{106}$ Ainsi dans le cas du soulèvement des étudiants chinois en 1989. Le pouvoir bloque la désectorisation par un traitement différentiel des étudiants et des ouvriers. Ces derniers sont traditionnellement plus durement réprimés et ils sont cantonnés de force dans leurs usines pendant le mouvement. À quoi il faut ajouter le peu d'appétence
} 
L'ensemble des contributions réunies ici insiste sur l'importance du rapprochement momentané de groupes que tout sépare et différencie en conjoncture routinière. Islamistes et militants de gauche et d'extrême gauche; jeunes urbains et éduqués mais aussi jeunes « débrouillards », « bidonvillois » et « déclassés »; classes moyennes précarisées, mais aussi professions intellectuelles et entrepreneurs exclus du premier cercle du pouvoir. Même au Bahraïn, la mobilisation était sur le point de dépasser le clivage entre sunnite et chiite, lequel recoupe par ailleurs de profonds clivages socio-économiques, jusqu'à ce que son extension soit stoppée par l'intervention militaire de l'Arabie saoudite.

Les phénomènes de défection s'observent à plusieurs niveaux. En premier lieu, de nombreux analystes ont formulé des hypothèses concernant l'attitude de «l'armée » en Tunisie et en Égypte comme en Libye et en Syrie. Quatre déterminants de leur attitude en ressortent : le rapport aux gouvernants, l'implication dans la vie des affaires, le niveau de formation, l'ouverture aux armées occidentales ${ }^{107}$. Toutefois, une analyse minutieuse reste à faire pour tenir compte de l'hétérogénéité d'institutions jusque-là considérées comme des « appareils répressifs monolithiques » ${ }^{108}$, pour décoder, séquence par séquence, les processus qui ont sous-tendu les calculs à différents moments de la dynamique révolutionnaire et ce aux différents niveaux hiérarchiques. En second lieu, des chercheurs ont examiné les dissensions au sein des partis dominants, l'effritement de leurs réseaux clientélaires, les processus de ralliement des anciens soutiens du régime au mouvement de révolte (voir les contributions sur la Tunisie) ${ }^{109}$. L'observation de ces événements aurait conduit les autres régimes de la région à en tirer des leçons pour justement prévenir les divisions internes et la défection des militaires. Ces stratégies correspondraient à des « jeux complexes et multiniveaux ${ }^{110}$. Les régimes tentent d'acheter la paix sociale; ils produisent un discours en vue d' «affecter les calculs stratégiques des citoyens » en soulignant les risques de profond désordre social (fitna), ils organisent des manifestations de soutien pour contrer les effets d'entraînement; ils dénoncent le caractère sectoriel voire manipulé par l'étranger des manifestations; ils observent les stratégies diplomatiques dans la région et se réajustent en conséquence. Au Maroc par exemple, outre ces stratégies, le régime propose une offre de réforme susceptible de réacheminer les acteurs « égarés » vers les canaux de la politique instituée ; il multiplie les négociations officielles et officieuses, les pressions individuelles et collectives; il réactive les réseaux des élus locaux ; il module ses options répressives d'une séquence de la dynamique protestataire à l'autre, tout en s'ajustant aux inflexions qu'il perçoit sur le plan régional et international (Bennani-Chraïbi, Jeghllaly). Au Yémen, le régime tente de compenser les premières défections en se constituant de nouveaux soutiens intérieurs et en remobilisant ses alliés extérieurs traditionnels, non sans brandir la menace sécuritaire (Bonnefoy, Poirier).

La notion de «modularité » permet également de pointer l'une des propriétés de l'extension des mobilisations "'. Elle désigne la diffusion des modes d'action et des cadrages au sein d'une unité nationale et éventuellement - comme dans le cas des « révoltes arabes »de pays à pays. La modularité est favorisée par l'émulation, soit lorsque le succès d'une performance laisse à penser que les chances de succès sont bonnes. Mais encore faut-il qu'il y

des étudiants pour la jonction des luttes. Voir Elizabeth J. Perry, «Intellectuals and Tiananmen. Historical Perspective on an Aborted Revolution », dans Daniel Chirot (ed.), The Crisis of Leninism and the Decline of the Left. The Revolutions of 1989, Seattle, University of Washington Press, 1991, p. 129-146. Sur les effets ambivalents de la répression sur les groupes contestataires comme sur les individus, voir Hélène Combes, Olivier Fillieule, «De la répression dans ses rapports à l'activité protestataire. Modèles structuraux et interactions stratégiques », Revue française de science politique, 61 (6), décembre 2011, p. 1047-1072.

107 Jean-François Coustillière, «Les forces armées dans les révoltes arabes », Confluence Méditerranée, 11, 2011, p. 67-80.

108 V. Geisser, A. Krefa, «L'uniforme ne fait plus le régime... », art. cité, p. 94. Voir aussi Anne Alexander, « Brothers-in-Arms? The Egyptian Military, the Ikhwan and the Revolutions of 1952 and 2011 », The Journal of North African Studies, 16, 2011, p. 533-554.

109 Par exemple, Sarah Ben Néfissa, «Ces 18 jours qui ont changé l'Égypte. Révolution civile et politique », Revue Tiers Monde, hors série, 2011, p. 227-236, dont p. 231. Sur le processus de transformation d'élites locales en contre-élites, voir aussi Guilain Denoeux, Urban Unrest in the Middle East, Albany, State University of New York Press, 1993.

110 Steven Heydemann, Reinoud Leenders, «Authoritarian Learning and Authoritarian Resiliance : Regime Responses to the "Arab Awakening" ", Globalizations, 8 (5), 2011, p. 647-653, dont p. 648.

"Sidney Tarrow, Democracy and Disorder, Oxford, Oxford University Press, 1989. 
ait « attribution de similarité ${ }^{\prime \prime 2}$. Celle-ci est produite par des caractéristiques institutionnelles ou culturelles communes ou perçues comme telles, ce que montre El Chazli dans sa contribution à propos de l'appel à la manifestation du 25 janvier dans le sillage de la chute rapide de Ben Ali, mais aussi par la manière dont les potentats de la région ont su tirer les leçons des coups heureux ou malheureux joués par leurs voisins dans les tentatives de retour au calme.

Le cas des « révoltes arabes » montre combien l'attribution de similarité est largement le produit d'un travail politique de la part d'agents de courtage occupés à l'unification des perceptions et à la mise en sommeil au moins provisoire des différends sur la méthode et les objectifs ; travail facilité par la forme « campement », sur laquelle nous reviendrons plus bas, mais aussi par la circulation des slogans, à la Kasbah de Tunis ${ }^{113}$ ou sur Tahrir (El Chazli). Dans les rues de Casablanca, l'observation de la morphologie des manifestations dénote une attention serrée à ce travail d'unification : comités de coordination pour la détermination des slogans, de la communication, de la logistique - dont bien sûr le dispositif de sonorisation - et le service d'ordre (Bennani-Chraïbi, Jeghllaly). Au Yémen également, des efforts sont menés pour en rester à un niveau de généralité qui autorise tous les investissements de sens et permet de maintenir des appartenances et des identités demeurées fortes et visibles (Bonnefoy, Poirier). Ce travail d'unification politique ne se voit jamais aussi bien qu'au moment où il se délite, comme lorsque de rares étudiants islamistes entonnent la ilaha illa Allah le 14 janvier à Tunis pour contrer les slogans gauchistes (Allal), ou encore lorsque par exemple, après le départ de Moubarak, les Frères musulmans et les Salafistes se rapprochent des militaires : la place Tahrir devient alors un lieu de confrontation entre groupes séculiers et « religieux », entre révolutionnaires «modérés» partisans d'un retour au calme et révolutionnaires « radicaux » favorables à la poursuite du mouvement de contestation jusqu'à ce que l'armée retourne dans ses baraquements ${ }^{114}$. Toutefois, reconnaître le travail politique d'unification ne doit pas conduire à un biais stratégiste et M. Béchir Ayari souligne, dans le cas tunisien, que la réduction des griefs à un commun dénominateur («dignité » et « dégage! ») n'est pas qu' « intentionnel» et s'inscrit dans une histoire plus longue des conflits sociaux politiques dans le pays ${ }^{115}$.

\section{Le troisième homme}

Rod Aya pointe non sans ironie combien la sociologie des révolutions, bien qu'elle dénonce rituellement la théorie du choix rationnel, ne dispose pas d'une théorie générale alternative de l'action humaine et que même dans les modèles les plus structuralistes des révolutions, les acteurs sont à un moment ou à un autre convoqués pour rendre compte du « passage à l'acte », faisant rentrer par la fenêtre ce que l'on avait chassé à grand bruit par la porte ${ }^{116}$.

L'on ne s'étonnera pas dans ces conditions de ce que la littérature oscille sans cesse entre des couples d'opposition peu convaincants : le duo spontanéité des foules et contagion des émotions versus action calculatrice et manipulatrice des meneurs ${ }^{11}$; l'opposition entre prévalence des choix tactiques et de l'invention, d'un côté, et repli sur les formes apprises

\footnotetext{
12 Doug McAdam, « Pour dépasser l'analyse structurale de l'engagement militant », dans Olivier Fillieule (dir.), Le désengagement militant, Paris, Belin, 2005, p. 49-74. Le mécanisme de l'attribution de similarité implique que l'information seule ne suffit pas à l'adoption d'une nouvelle idée ou d'une pratique. Pour qu'il y ait diffusion et donc imitation, il faut qu'il y ait identification minimale des imitateurs potentiels aux initiateurs.

${ }^{113}$ Choukri Hmed, "Si le peuple un jour aspire à vivre, le destin se doit de répondre" », Les Temps modernes, 664, 2011, p. 4-20.

114 Khaled Dawoud, «Tahrir Countershow of Force», Al Ahram Weekly, 11-17 août 2011, <http://weekly.ahram.org.eg/2011/1060/fr1.htm>.

"1s Michaël Béchir Ayari, «Des maux de la misère aux mots de la "dignité" », Revue Tiers Monde, hors série, 2011, p. 209-218, dont p. 216.

${ }^{116}$ R. Aya, « The Third Man... », art. cité.

${ }^{11}$ Sur les approches en termes de contagion des émotions, voir la synthèse critique de Susanna Barrows, Miroirs déformants. Réflexions sur la foule en France à la fin du $19^{e}$ siècle, Paris, Aubier, 1990. Sur le couple foules manipulées et meneurs manipulateurs, notamment dans les catégories de l'entendement policier, voir Olivier Fillieule, Stratégies de la rue. Les manifestations en France, Paris, Presses de Sciences Po, 1997.
} 
dans les situations d'incertitude structurelle de l'autre ${ }^{118}$.

L'ambiguïté systématique se nourrit à la fois de la difficulté qu'il y a à percevoir le caractère structuré des comportements en foule ${ }^{19}$ et de l'ambivalence des discours tenus par les témoins et les acteurs eux-mêmes ${ }^{120}$. Dans son analyse des sit-in du mouvement des droits civiques au début des années 1960, F. Polletta en offre une frappante illustration, entre sentiment de spontanéité et d'imprévisibilité et reconnaissance du rôle moteur des leaders et de la préparation des actions ${ }^{121}$. Aussi bien dans les « révoltes arabes", les exemples abondent, qu'il s'agisse d'interviews de participants, ou encore de reportages filmés et diffusés par les media arabes et internationaux ${ }^{122}$.

La notion développée par M. Dobry d' «évasion des calculs », qui se tient à égale distance entre l'inertie des systèmes de disposition et la rupture d'intelligibilité nous semble ici très utile. En s'appuyant sur la notion «d'anticipation mutuelle » chez Goffman "23, M. Dobry avance que dans les phases de désectorisation généralisée où l'incertitude structurelle prévaut, les acteurs se fondent sur l'information que leur livre l'attitude des autres acteurs pour déployer des stratégies, le tout dans un cadre normatif structurellement déterminé mais déréglé et en voie d'effondrement partiel ou total ${ }^{124}$. "L'invention, dans cette perspective, n'est en somme ni la duplication pure et simple de ce qui a été intériorisé, ni le surgissement soudain et mystérieux, ex nihilo, de l'innovation. » ${ }^{125}$

Admettre cela implique de ne pas se détourner trop vite des approches rational choice, auxquelles il faut reconnaître d'avoir établi que l'action des individus est le produit conjoint d'un choix rationnel (ce qui cependant ne dit rien sur ce qui motive ces choix) et de l'espoir de succès (ce qui ne présuppose pas du caractère « raisonnable » de ces choix) ${ }^{126}$. À partir de ces deux postulats que nous partageons entièrement, il est possible de réfléchir aux microfondations de l'action révolutionnaire et aux mécanismes d'agrégation en situation d'incertitude.

La littérature s'inscrivant dans le périmètre du rational choice suggère un certain nombre de processus propres aux situations révolutionnaires qui nous aident, sous une forme idéale-typique, à comprendre comment les acteurs calculent. L'on pense tout particulièrement à la théorie dite de la masse critique, qui prédit que la décision de s'engager dans l'action

\footnotetext{
${ }{ }^{\prime}$ Michel Dobry a souligné combien l'« illusion héroïque » était prégnante dans les travaux sur les situations de crise, et les impasses auxquelles elle conduisait. La critique peut s'étendre à un certain nombre de conceptions de l'événement comme «rupture d'intelligibilité » ouvrant une situation dans laquelle l'invention domine et s'affranchit de toute détermination structurelle. Par exemple : Alban Bensa, Éric Fassin, « Les sciences sociales face à l'événement », Terrain, 38, 2002, p. 5-20.

${ }^{119}$ Clark McPhail, The Myth of the Madding Crowd, New York, Aldine/De Gruyter, 1991, p. 158-84 ; Olivier Fillieule, «The Independent Psychological Effects of Participation in Demonstrations », Mobilization. An International Journal, 17 (3), 2012, p. 489-502.

${ }^{120}$ L'on pense bien sûr aux réflexions d'Emmanuel Kant sur l'enthousiasme révolutionnaire dans Le conflit des facultés (11' section). Voir aussi, pour s'en tenir à la révolution française, les déclarations d'acteurs reproduites et analysées dans Michel Vovelle, La mentalité révolutionnaire, Paris, Messidor/Éditions sociales, 1985, ou encore les pages que Timothy Tackett consacre à la nuit du 4 août 1789, marquée par « l'enthousiasme et un esprit de sacrifice remarquables », dans Becoming a Revolutionnary. The Deputies of the French National Assembly and the Emergence of a Revolutionnary Culture (1789-1790), Princeton, Princeton University Press, 1996, p. 167.

${ }_{121}$ Francesca Polletta, “"It was Like a Fever...". Narrative and Identity in Social Protest », Social Problems, 45 (2), mai 1998, p. 137-159.

${ }_{122}$ À titre d'exemple, le beau film de Stephano Savona (Tahrir, 2011) et les témoignages publiés dans Hatem Rushdy, 18 days in Tahrir. Stories from Egypt's Revolution, Hong Kong, Haven Books, 2011.

${ }^{123}$ Erving Goffman, Strategic Interaction, Oxford, Basil Blackwell, 1970, p. 85-145.

${ }^{124}$ Dans son livre sur les «abdications collectives», Ivan Ermakoff fournit une analyse proche sous certains aspects des processus de décision dans de telles situations de fluidité politique, à partir de la théorie des jeux (et donc sans se soucier d'associer l'explication cognitive à l'analyse contextuelle). Il développe notamment l'idée selon laquelle, dans ces situations chargées d'enjeux et dans lesquelles, jusqu'au dernier moment, l'issue apparaît incertaine, les processus d'alignements entre les différents acteurs constituent un facteur explicatif décisif. Voir Ivan Ermakoff, Ruling Oneself Out. A Theory of Collective Abdications, Durham, Duke University Press, 2008 (cf. infra le compte rendu de ce livre, p. ?-?).

${ }^{125}$ M. Dobry, Sociologie des crises politiques, op. cit., p. 260.

${ }^{126}$ R. Aya, «The Third Man...», art. cité, p. 148. On sait en effet depuis les travaux de Evans Pritchard à quel point la rationalité de l'action est toujours relative à des cadres de référence cognitifs. Voir Evans Pritchard, Witchcraft, Oracles and Magic among the Azande, Oxford, Oxford University Press, 1937.
} 
dépend de l'implication attendue d'un nombre suffisant de personnes ${ }^{127}$ et s'articule à plusieurs autres résultats ${ }^{128}$ dont notamment les propositions sur la disjonction entre préférences privées et préférences publiques dans les États autoritaires, renforcée par la peur de la répression ${ }^{129}$. Du coup, lorsqu'une situation révolutionnaire se déclenche et que les individus ont le sentiment que leurs préférences privées concernant le changement de régime sont partagées par un grand nombre d'autres, ils peuvent, passé un certain seuil ${ }^{130}$, s'engager très vite et massivement, apportant une explication aux phénomènes de «revolutionary bandwagon ${ }^{131}$.

De nombreux témoignages illustrent ce phénomène. Pour A. El Aswany, le 25 janvier est un jour comme un autre... jusqu'en milieu de journée. En allumant la télévision, le romancier constate qu'un «miracle » s'est produit, que les Égyptiens se sont massivement mobilisés contre le régime ${ }^{132}$. Dès lors, il s'habille précipitamment pour rejoindre la place Tahrir. Dans sa contribution à ce dossier, Y. El Chazli montre que le décalage perçu par les «novices » entre l'ampleur de la mobilisation du 25 janvier et la confidentialité des sit-in habituels les galvanise. C. Hmed indique qu'à Sidi Bouzid, les effets mobilisateurs du discours de Ben Ali le 13 janvier l'emportent sur ses effets démobilisateurs, précisément par ce phénomène d'anticipation. De même, au Maroc, le processus lancé par les pionniers du Mouvement du 20 février et, préalablement par les pionniers du «Printemps arabe », suscite des ralliements une fois les premiers succès engrangés. Inversement, comme le montrent M. Bennani-Chraïbi et M. Jeghllaly dans ce dossier, le reflux du mouvement coïncide avec une sorte de contre «bandwagon effect» : le sentiment que le mouvement a perdu la bataille, la perception d'une décélération de l'histoire contribuent à accélérer la démobilisation.

Au-delà des mécanismes qu'elle pointe de manière stylisée, cependant, l'approche rational choice est d'un faible secours pour restituer la manière dont les acteurs calculent en situation. En premier lieu, ce qui l'intéresse d'abord est de prédire l'engagement, à partir de l'identification de préférences préalables. Ici, c'est tout ce qui fait l'épaisseur et la dynamique des événements qui échappe à l'analyse ${ }^{133}$. En effet, les individus ajustent leurs calculs en fonction de matrices de coûts/bénéfices indexées sur des contextes fortement volatils et c'est justement de cela dont il faut rendre compte. De ce point de vue, T. Tackett offre une approche des calculs en situation révolutionnaire infiniment plus convaincante. Il reconstruit avec minutie les voies par lesquelles les députés aux États généraux sont progressivement devenus révolutionnaires, ce qui implique d'étudier la transformation de leurs valeurs et de leurs modes de pensée en s'intéressant à toutes les dimensions de leur existence, soit aussi bien leurs idées que leurs soucis matériels tels qu'ils s'expriment notamment dans leurs courriers privés ${ }^{134}$. Dans sa contribution à ce numéro, Youssef El Chazli offre une illustration de la façon dont naissent progressivement sur la place Tahrir, une psychologie et une culture révolutionnaires.

En second lieu, l'anthropologie au fondement de la théorie du choix rationnel la cantonne le plus souvent à un certain cognitivisme. Cela, même lorsque des tentatives sont

\footnotetext{
${ }^{127}$ Gerard Marwell, Pamela Oliver, The Critical Mass in Collective Action. A Micro-Social Theory, Cambridge, Cambridge University Press, 1993. Cette théorie prend l'exact contre-pied du paradoxe olsonien, sans l'invalider cependant puisqu'elle entend s'appliquer aux seules situations de forte incertitude.

${ }^{128}$ Voir Mark I. Lichbach, The Rebel's Dilemma, Ann Arbor, University of Michigan Press, 1995, pour une revue très complète.

${ }_{129}$ Timur Kuran, Private Truths, Public Lies. The Social Consequences of Preference Falsification, Cambridge, Harvard University Press, 1995.

${ }^{130}$ Mark Granovetter, Roland Soong, «Threshold Models of Diffusion and Collective Behavior », Journal of Mathematical Sociology, 9, 1983, p. 165-179; Thomas C. Schelling, « Hockey Helmets, Concealed Weapons and Daylight Saving », Journal of Conflict resolution, 17 (3), 1973, p. 381-428.

${ }_{131}$ Sur les notions de «bandwagon», de «jeu d'assurance» et de «dé-assurance», voir Dennis Chong, Collective Action and the Civil Rights Movement, Chicago, The University of Chicago Press, 1991, et Rasma Karklins, Roger Petersen, «Decision Calculus of Protesters and Regimes: Eastern Europe 1989 », The Journal of Politics, 55 (3), août 1993, p. 588-614.

${ }_{132}$ Alaa El Aswany, Chroniques de la Révolution égyptienne, Paris, Actes Sud, 2011, p. 13.

${ }^{133}$ Charles Kurzman, «Can Understanding Undermine Explanation? The Confused Experience of Revolution », Philosophy of the Social Sciences, 34 (3), septembre 2004, p. 328-351.

${ }^{134}$ T. Tackett, Becoming a Revolutionnary..., op. cit.; voir également, dans un tout autre contexte, Christopher R. Browning, Ordinary Men. Reserve Police Battalion 101 and the Final Solution in Poland, New York, Harper Collins, 1992.
} 
menées pour « contextualiser » les modèles explicatifs : on reste toujours très loin d'une prise en compte suffisante des ancrages socioculturels des acteurs, soit de la nature et de la force des liens préexistants, des opportunités et des obligations qu'ils déterminent, de leurs ancrages spatiaux dans les matrices de coûts/bénéfices ${ }^{135}$. Qui plus est, et l'on rejoint là la critique précédente, de très nombreux travaux d'historiens ont montré comment la dynamique des épisodes émeutiers ou révolutionnaires contribuent aussi largement à redéfinir les liens communautaires et les formes de l'attachement interindividuel, rendant illusoire de chercher à reconstruire des matrices de coûts/avantages aux moyens de modèles statiques et unidimensionnels ${ }^{136}$.

En s'attachant à décrire de la manière la plus précise possible les dilemmes pratiques dans lesquels les acteurs sont pris à chaque moment du temps, en tâchant de reconstruire leurs motifs au plus près de ce qu'ils font et de ce qu'ils pensent, l'on peut espérer replacer au centre de l'explication le « troisième homme » qui manquait à l'appel ${ }^{137}$, l'acteur qui agit pour de «bonnes raisons », que ces raisons soient rationnelles ou pas aux yeux du chercheur et du monde, qu'elles soient peu liées, voire éloignées ou situées à l'opposé des causes qu'elles contribuent à servir.

\section{Performances révolutionnaires}

Sous les dehors de l'imprévisibilité et de l'effervescence collective, les événements sont paradoxalement inscrits dans des normes et se nourrissent de modes d'action puisés au sein de répertoires qu'il convient de décrire le plus finement possible. Dire cela ne revient pas à nier le caractère potentiellement transformateur des événements caractéristiques des situations révolutionnaires. Au contraire, il nous semble que l'on ne comprend jamais aussi bien les règles présidant à la plasticité des structures qu'à partir du moment où l'on reconnaît « leur sensibilité aux mobilisations, à l'activité tactique des acteurs, aux coups échangés » ${ }^{138}$. Dans notre perspective, si les situations révolutionnaires correspondent bien à des échanges contraints par la logique interactionnelle, il faut cependant prêter attention à la manière dont les conduites observables s'instituent historiquement, puisque les normes sociales qui s'y déploient ont fait et font l'objet d'élaborations progressives, multiples et concurrentes ${ }^{139}$.

Ces remarques invitent à observer de près les performances, lesquelles sont soumises à deux logiques en tension. D'un côté, les acteurs - protestataires et forces de l'ordre s'engagent le plus souvent dans des séquences d'interaction structurellement réglées ; cellesci indiquent le maintien d'un sens partagé de la situation, donc de règles du jeu explicites et implicites (encadrement légal, usages), nourries notamment d'une histoire manifestante et de cultures protestataires et policières, avec ses gestes attendus et ses coups prévisibles ${ }^{100}$. De l'autre, la multiplicité des acteurs en présence et la complexité des luttes de sens qui se jouent

\footnotetext{
${ }_{135}$ Roger V. Gould, Insurgent Identities. Class, Community, and Protest in Paris from 1848 to the Commune, Chicago, The University of Chicago Press, 1995 ; Karl Dieter Opp, Peter Voss, Christiane Gern, Origins of $a$ Spontaneous Revolution, Ann Arbor, Michigan University Press, 1995 ; Steven Pfaff, «Collective Identity and Informal Groups in Revolutionary Mobilization. East Germany in $1989 »$, Social Forces, 75 (1), 1996, p. 91118.

${ }^{136}$ Par exemple, William M. Reddy, «The Textile Trade and the Language of the Crowd at Rouen, 1752-1871 », Past and Present, 74 (1), 1977, p. 62-89, dont p. 82-83 ; Cynthia A. Bouton, The Flour War. Gender, Class, and Community in Late Ancien Régime French Society, Philadelphie, Penn State Press, 1993, chap. 5.

${ }^{137}$ R. Aya, « The Third Man... », art. cité.

${ }^{138}$ Michel Dobry. "Le politique dans ses états critiques. Retour sur quelques aspects de l'hypothèse de continuité », dans Marc Bessin, Claire Bidart, Michel Grossetti (dir.), Bifurcations. Les sciences sociales face aux ruptures et à l'événement, Paris, La Découverte, 2010, p. 64-88, dont p. 79-80. Sur les rapports entre structure et événement, voir aussi Marshall Sahlins, How «Natives » Think. About Captain Cook, for Example, Chicago, The University of Chicago Press, 1995 ; William H. Sewell Jr., Logics in History. Social Theory and Social Transformation, Chicago, Chicago University Press, 2005. Plus récemment, voir Stéphane Latté, «La force de 1'“événement" est-elle un artefact ? », Revue française de science politique, 62 (3), juin 2012, p. 409432 .

${ }^{139}$ Erving Goffman, Behavior in Public Places, Free Press of Glencoe, 1963, p. 12 ; C. McPhail, The Myth of the Madding Crowd, op.cit., p. 158-184; Nicolas Mariot, «Does Acclamation Equal Agreement? Rethinking Collective Effervescence Through the Case of the Presidential "Tour de France" during the 20" Century ", Theory \& Society, 40 (2), mars 2010, p. 191-221; Olivier Fillieule, Danielle Tartakowsky, Street Demonstrations, Winnipeg, Fernwood Publishing, 2012.

${ }_{140} \mathrm{O}$. Fillieule, Stratégies de la rue..., op. cit.
} 
dans l'interaction et son interprétation introduisent de fortes marges d'incertitude. Autrement dit, seule l'analyse contextualisée de l'échange de coups en situation permet de comprendre selon quelles logiques la dramaturgie manifestante peut instaurer et orienter une situation révolutionnaire, pour peu que l'on tienne compte des mouvements des protestataires autant que de ceux de l'autorité contestée et des forces de répression sur le terrain.

Les spécialistes des mouvements sociaux en Afrique du Nord et au Moyen-Orient ont noté combien les conduites observables dans les manifestations sont historiquement instituées et combien les normes sociales qui s'y déploient s'inscrivent dans des savoir-faire et des savoir être élaborés dans les luttes passées, directement réutilisés par les militants ou transmis aux plus jeunes ${ }^{141}$. Que ce soit en Tunisie, en Égypte, au Maroc ou au Yémen, le tour que prend la dynamique protestataire doit être rapporté aux mobilisations antérieures : les actions de soutien aux causes «arabes » ou «islamique»; les mobilisations inédites comme à Redeyef et Gafsa en 2008, à Ben Guerdane en 2010, ou la vague de grèves ouvrières qui enfle en Égypte à partir de 2004 ; les coordinations contre la vie chère comme au Maroc jusqu'en 2009 ; le développement de groupes transcendant les clivages idéologiques (à l'instar de Kifaya, du groupe du 6 avril, et de l'Association nationale pour le changement en Égypte, des organisations de droits humains dans ces différents pays). Ces mobilisations permettent des jonctions partielles entre des acteurs appartenant à des réseaux sociopolitiques concurrents (voir les contributions de Bennani-Chraïbi et Jeghllaly, de Hmed et de El Chazli). Elles favorisent l'apprentissage de la protestation collective par des millions de personnes. Elles amènent les militants aguerris à apprendre de leurs échecs précédents, à innover pour mieux surprendre les forces de l'ordre (voir Hmed; El Chazli ; Bennani-Chraïbi, Jeghllaly). Il ne faut pas non plus négliger les savoir-faire accumulés et les réseaux informels constitués par les Ultras des clubs de football ou plus globalement dans les lieux de dissidences urbaines (voir Allal) ${ }^{142}$. Enfin, il importe de prendre en compte tout ce qui s'apprend d'un mouvement à l'autre (y compris de façon transnationale), tant du côté des protestataires que des forces de l'ordre.

Mais les comportements des acteurs ne s'inscrivent pas uniquement dans les formes apprises ; ils dépendent aussi des logiques de la situation, marquées par la profusion de moments critiques. On le voit bien au travers des effets ambivalents de la répression qui peut radicaliser les opposants en instaurant des codes binaires ${ }^{143}$, lesquels accélèrent l'effondrement de la légitimité du pouvoir et poussent les participants à aller bien au-delà des objectifs initialement assignés (voir Allal et Hmed), alors même que pour le pouvoir, la violence indiscriminée et la sauvagerie de la répression visaient à réduire la mobilisation.

$\mathrm{Ce}$ phénomène est particulièrement visible dans toute une série d'événements cathartiques qui jouent le rôle de «turning points », à l'instar du «Vendredi de la Colère », le 28 janvier 2011 en Égypte (El Chazli), marqué par la disparition des forces de sécurité, le rôle ambigu de l'armée, la mise sur pied des comités d'autodéfense dans les quartiers ${ }^{14}$. Au Yémen, le 18 mars 2011, avec le «Vendredi de la Dignité », les forces de sécurité interviennent pour déloger les protestataires de la place Taghyir et plus de cinquante personnes sont assassinées par des tireurs isolés, ce qui galvanise la mobilisation et accélère le rythme des défections. À Manama, si les premières manifestations à partir du 14 janvier 2011 ne réunissent que quelques centaines de personnes, la répression qu'elles suscitent joue un rôle mobilisateur. L'installation d'un campement sur la place de la Perle et la répression qui s'ensuit transforme la nature de la contestation sous deux aspects: les demandes se radicalisent, la revendication de réformes disparaissant au profit de l'exigence d'un changement de régime; la protestation jusqu'alors surtout cantonnée aux militants des droits humains ${ }^{145}$ s'étend aux étudiants, aux ingénieurs, professeurs et avocats, mais aussi aux

\footnotetext{
${ }^{1+1}$ Mounia Bennani-Chraïbi, Olivier Fillieule (dir.), Résistances et protestations dans le monde musulman, Paris, Presses de Sciences Po, 2003; Joel Beinin, Frédéric Vairel (eds), Social Movements, Mobilization and Contestation in the Middle East and North Africa, Stanford, Stanford University Press, 2011. Il en va de même des forces de maintien de l'ordre, lesquelles conservent également une mémoire des luttes passées.

${ }_{142}$ Ashraf El-Sherif, «The Ultras' Politics of Fun Confront Tyranny», Jadaliyya, février 2012, $<$ http://www.jadaliyya.com/pages/index/4243/the-ultras-politics-of-fun-confront-tyranny $>$.

${ }_{143}$ J. C. Alexander, Performative Revolution in Egypt, op. cit., p. 3.

${ }_{144}$ J. Gelvin, The Arab Uprisings..., op . cit., p. 46 ; H. Rushdy, 18 days in Tahrir..., op. cit.

${ }^{145}$ Par exemple, Preethi Nallu, «A Family under Siege. The Khawajas in Bahrain », Jadaliyya, mars 2012, $<$ http://www.jadaliyya.com/pages/index/4738/a-family-under-siege_the-khawajas-in-bahrain $>$.
} 
acteurs de l'opposition, aux syndicalistes et leaders religieux chiites, marquant une extension de la contestation à de nombreuses couches de la société. Ces quelques exemples illustrent la dynamique autonome des événements transformateurs. C'est la force des événements euxmêmes qui entraîne les participants bien au-delà de tout ce qu'ils avaient pu initialement imaginer, voire rêver (Allal ; Hmed; El Chazli). Et si les manifestants de Sidi Bouzid, de la Kasbah de Tunis, de la place Tahrir, de Deraa sont remarquablement conscients de la signification historique de leur action, ils restent cependant incapables de l'expliquer tant les logiques de la situation dépassent leurs calculs individuels et leurs attentes.

Le recours à une pièce du répertoire d'action plutôt qu'à une autre, dans un univers donné de contraintes, répond à la fois à des considérations tactiques et stratégiques, et à une activité de cadrage déployée par les contestataires pour imposer le sens de leur mouvement. Pendant les soulèvements arabes, l'on observe une hétérogénéité de modes d'action : grèves générales, manifestations géantes, funérailles politiques, rassemblements et émeutes, mais aussi actions de guérilla. Cette hétérogénéité est indexée sur les formes de politisation des acteurs, sur leurs réseaux préexistants, les lieux qu'ils investissent, le timing qu'ils choisissent pour se mobiliser. Les contributions de ce dossier sur la Tunisie l'illustrent bien. Les « jeunes débrouillards » se coordonnent à travers des relations de voisinage, amicales et familiales et tendent à se livrer (plutôt la nuit) à une guérilla urbaine contre les forces de police dans les ruelles de leurs quartiers. En revanche, les syndicalistes et les opposants politiques se mobilisent à travers des réseaux militants préexistants, ils organisent (pendant la journée) des actions tributaires des leçons du passé, à l'instar des grèves générales, des sit-in, des caravanes de solidarité ou des manifestations dans les grandes artères du centre-ville. Une telle hétérogénéité n'exclut pas pour autant les jonctions.

À titre d'exemple, dans les soulèvements tunisien, égyptien, bahreïni, libyen et yéménite, l'ensemble de ces dynamiques converge au sein de l'espace concret du campement, autorisant à interpréter ce mode d'action comme un efficace opérateur d'extension de la mobilisation ${ }^{146}$. La place Tahrir au Caire en est l'illustration la plus éloquente ${ }^{147}$ : la « révolution égyptienne » y prend symboliquement corps et son destin tout entier y semble suspendu ${ }^{148}$. Phénomène que l'on retrouve à des degrés divers à la Kasbah de Tunis, sur la place du Taghyir à Sanaa, sur la place de la Perle à Manama mais aussi à Benghazi sur AlChajara, à Tripoli sur la Place verte, etc. ${ }^{49}$. Dans les contextes non démocratiques où la participation à une manifestation de rue peut être très coûteuse pour soi et pour ses proches, le campement est à la fois disruptif symboliquement et particulièrement ajusté aux contraintes posées par la répression (voir les contributions sur l'Égypte et le Yémen). En premier lieu, il permet d'instaurer des espaces «libérés », qui aident à surmonter la peur, à inscrire le mouvement dans la durée, un peu sur le modèle plus ancien de la barricade ${ }^{150}$. C'est aussi un moyen de construire une image unitaire du mouvement, comme reposant sur une levée en masse de toutes les classes de la société contre un pouvoir illégitime ${ }^{151}$ et surtout comme pacifique. Enfin, comme le montrent El Chazli, Bonnefoy et Poirier dans ce dossier, le campement est un puissant dispositif favorisant la fabrique d'appétences révolutionnaires,

\footnotetext{
146 Voir la conclusion à Olivier Fillieule, Danielle Tartakowsky, Street Demonstrations, op . cit ., à paraître.

${ }^{47}$ Ce mode d'action est si prépondérant que cela a pour effet d'éclipser aux yeux des commentateurs, et peut-être aussi jusqu'à un certain point des acteurs, les autres modes d'action. Aussi bien, les regards se sont focalisés très vite sur les capitales, délaissant ainsi la contribution d'autres villes à la dynamique de la contestation.

${ }_{148}$ Dans les témoignages recueillis dans 18 days in Tahrir les personnes interrogées sont intimement persuadées que tout se joue sur la place et nulle part ailleurs en Égypte.

${ }_{149}$ Ce mode d'action ne s'invente pas à la Kasbah de Tunis ni sur la place Tahrir et serait redevable d'une histoire. L'exemple qui vient d'abord à l'esprit est celui de la place Tien An Men en 1989, mais aussi celui des mouvements contemporains (Indignés et Occupy en Europe, aux USA et en Israël). Par ailleurs, ces espaces ne deviennent pas des épicentres de la contestation du jour au lendemain; à l'instar de la place Tahrir au Caire ou de la place Mohammed V à Casablanca (place Lahmam), ils ont souvent été constitués comme des lieux symboliques de l'activisme bien avant 2011.

${ }^{150}$ Sur la nature et le sens de la barricade, voir Alain Corbin, Jean-Marie Mayeur (dir.), La Barricade, Paris, Publications de la Sorbonne, 1997 ; Mark Traugott, The Insurgent Barricade, Berkeley, University of California Press, 2010. Pour une synthèse, voir Michel Offerlé, « The barricade », dans David A. Snow, Donatella Della Porta, Bert Klandermans, Doug McAdam (eds), The Wiley-Blackwell Encyclopedia of Social and political Movements, Oxford, John Wiley, 2013, à paraître.

${ }_{\text {sI }}$ Ici, ce que J. Jasper nomme « the power of negative thinking » joue à plein (The Art of Moral Protest, Chicago, The University of Chicago Press, 1997, p. 362).
} 
notamment grâce au renforcement de l'esprit de corps au sein des petits groupes ${ }^{152}$. Deux éléments sont là cruciaux.

D'une part la participation aux campements, par la durée qu'elle implique et la nature des échanges qui s'y déploient, a toutes les chances d'avoir plus d'effets que la simple participation à des manifestations ponctuelles, même violentes. De cela, de très nombreux éléments témoignent, au premier rang desquels les messages Facebook et le fil des messages twitter postés à chaud pendant les événements. Ceux-ci - au-delà des obstacles à leur recueil et à leur traitement - constituent un matériau exceptionnel pour qui voudrait reconstituer heure par heure l'évolution de l'état d'esprit des participants, leurs perceptions, leurs calculs et pour le dire d'un mot «l'émergence d'une norme situationnelle» ${ }^{153}$.

D'autre part, l'intensification des contacts interpersonnels, des débats contradictoires et des discussions tout comme la «libération de la parole politique » ${ }^{154}$ font écho aux observations des historiens de la Révolution française lorsqu'ils scrutent la mentalité révolutionnaire ${ }^{155}$. À la Kasbah et sur Tahrir, comme au Palais Royal au début des années 1790 à Paris, des individus s'improvisent orateurs et haranguent de petits groupes, des rondes de débat (halaqat niqashiyya) se forment sur la base de réseaux préalables d'interconnaissance mais auxquels peuvent s'agréger d'autres petits groupes. L'espace saturé de signes politiques (drapeaux, pancartes, graffitis) est aussi un espace où l'on chante, où l'on danse, où l'on s'amuse et partage de la nourriture (voir El Chazli) ${ }^{156}$. Le campement et les manifestations qui en rythment l'existence permettent «d'assister ou de participer à la naissance de nouvelles pratiques, de nouveaux objets, d'un nouveau cadre, qui trahissent une transformation plus ou moins radicale des us et coutumes et contribuent à la formation d'une identité nouvelle » ${ }^{15}$. Les initiations aux savoir-faire se font dans plusieurs sens. Les acteurs organisés issus des couches moyennes transmettent leurs expériences aux «novices »; de mềme « les bourgeois s'encanaillent, s'ouvrent de manière inédite à la culture populaire des jeunes urbains qui leur apprennent à se protéger et les initient aux moyens de se procurer des produits rares $»{ }^{158}$. Ces observations nous rappellent l'importance de la dimension spatiale dans l'analyse des mouvements sociaux, entendue ici à la fois comme une ressource, un enjeu de lutte et de mobilisation ${ }^{159}$, potentiellement générateur de sociabilité et de politisation (voir Hmed et Allal) 160 .

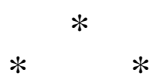

Dans ce texte nous avons défendu une approche réfutant tout causalisme et centrée sur les situations révolutionnaires, aux dépens de la recherche de facteurs déterminants ou d'éléments déclencheurs d'une part, et de la réflexion sur les conséquences, ou plutôt les résultats de ces situations, d'autre part. Non que la recherche de facteurs contextuels pour comprendre les dynamiques d'émergence nous paraisse superflue ${ }^{161}$, ni que la réflexion sur les

\footnotetext{
152 O. Fillieule, « The Independent Psychological Effects... », art. cité.

${ }_{153}$ Ralph H. Turner, Lewis M. Killian, Collective Behavior, Englewood Cliff, Prentice Hall, 1957.

${ }^{154} \mathrm{C}$. Kurzman, The Unthinkable Revolution in Iran, op. cit.

${ }_{155} \mathrm{M}$. Vovelle, La mentalité révolutionnaire, op. cit.

156 Voir aussi Iman Farag, «En marge de la révolution égyptienne : écrire l'histoire ou sauver la mémoire?», Mouvements, 66, 2011, p. 42-47 ; Iman Mersal, « Revolutionary Humor», Globalizations, 8 (5), 2011, p. 669674 ; Lila Abu-Lughod, "Living the "Revolution" in an Egyptian Village. Moral Action in a National Space », et Reem Saad, « The Egyptian Revolution. A Triumph of Poetry », American ethnologist, 39 (1), 2012, p. 21-25, et p. 63-66.

${ }^{15}$ Annie Jourdan, La Révolution, une exception française ?, Paris, Flammarion, 2004, p. 142.

158 Témoignage de la politiste Dina El-Khawaga recueilli par les auteurs.

${ }^{159}$ Par exemple, Choukri Hmed, «Espace géographique et mouvements sociaux », dans O. Fillieule, L. Mathieu, C. Péchu (dir.), Dictionnaire des mouvements sociaux, op. cit., p. 220-227.

${ }^{160}$ Asef Bayat, Street Politics. Poor People's Movements in Iran, New York, Cambridge University Press, 1997.

${ }^{161}$ Pour une mise au point critique sur la question de l'émergence, et de la combinaison de facteurs profonds externes et internes en même tant que de micro événements, voir Stéphane Cadiou, Stéphanie Dechezelles, «La problématique de l'émergence pour l'étude des mobilisations collectives: pistes et repères », dans Stéphane Cadiou, Stéphanie Dechezelles, Antoine Roger (dir.), Passer à l'action : les mobilisations émergentes, Paris, L'Harmattan, 2007, p. 11-48.
} 
conséquences soit sans intérêt ${ }^{162}$. Mais tout simplement parce que la part du lion a jusqu'à présent été réservée à ces deux aspects aux dépens de l'étude des situations elles-mêmes. La brève revue de la littérature que nous offrons en première partie de ce papier montre bien qu'il ne s'agit pas d'une vue de l'esprit.

Ce décentrement s'accompagne d'un autre déplacement tout aussi crucial. Les travaux portant sur les situations révolutionnaires ou plus généralement les situations de crise s'attachent d'abord à définir et à étudier les propriétés advenues de ces situations. La désectorisation, la constitution de coalitions interclassistes, la division ou la défection au sein des organes centraux de l'État et de l'appareil répressif, l'évasion des calculs, sont autant de résultats de l'ouverture d'une situation révolutionnaire. Nous proposons pour notre part de travailler sur les séquences d'action, définies comme des chaînes d'interaction complexes mais observables, conduisant (ou pas) à ces résultats. Deux voies s'ouvrent alors. L'on peut reconstituer les échanges de coup entre toutes les parties prenantes de ces performances, dans une situation révolutionnaire donnée, pour en isoler les faits les plus saillants et en retracer la dynamique. C'est dans cette perspective que s'inscrivent les papiers que l'on va lire ${ }^{163}$. L'on peut également, à partir de la mise en regard d'un certain nombre de situations révolutionnaires, et sans préjuger de leur réussite ou de leur échec, identifier dans les performances qui y conduisent une série de processus relationnels et cognitifs de portée relativement générale. L'on peut ce faisant approcher les modes de transformation d'états propres aux situations révolutionnaires. Nous l'avons souligné, une telle ambition n'est pas éloignée de celle qui anime les approches mécanistiques. Elle s'en distingue cependant par son déterminisme méthodologique et donc son rejet de tout causalisme, autant que par les échelles d'observation retenues, et tout particulièrement par son attachement aux microfondations de l'action. D'où notre insistance dans ce qui précède sur les acteurs et leurs calculs en situation, aussi bien que sur les échanges de coups et leur éventuelle saillance. Le tableau suivant offre une mise en ordre des éléments abordés plus haut, à partir d'une double distinction.

Distinction d'abord entre éléments conductifs, processus et situation révolutionnaire. Cette distinction diachronique entre trois classes d'éléments est bien entendu poreuse et redevable d'une spécification au cas par cas. En tout premier lieu parce que les processus qui conduisent aux situations révolutionnaires peuvent continuer d'y agir une fois celles-ci instaurées. Par ailleurs la distinction entre conditions de départ et composants des modes de changement d'état est difficile à opérer. Ce problème est classique pour les neuroscientifiques et les biologistes qui au fond tranchent sur la base de considérations pragmatiques ${ }^{164}$. Ce qui nous importe ici, et pour s'en tenir à un exemple, c'est de bien séparer par exemple au niveau environnemental la nature plus ou moins exclusive et interventionniste de l'Etat ${ }^{165}$ ou les formes prédominantes de segmentation sociale ${ }^{166}$, lesquelles cadrent en partie, au niveau micro et méso, la survenance, la forme et l'étendue des processus d'émulation et d'attribution de similarité, avec par exemple pour conséquence la modularité, soit la transférabilité accrue des modes d'action et des cadrages reconnaissables dans le temps et dans l'espace, ou l'extension de la contestation à une fraction significative de la population. Distinction ensuite entre trois échelles d'analyse qui n'est pas non plus sans poser des difficultés. Ainsi par exemple, le processus d'attribution de similarité (identification stratégique des imitateurs potentiels aux initiateurs) observable au niveau relationnel est lié, au niveau cognitif, à l'émulation (c'est-à-dire aux espoirs de succès). Malgré tout, la distinction nous semble utile aux fins de pointer des niveaux d'observation de ces processus, dont certains ne se réduisent

\footnotetext{
${ }^{162}$ Les travaux sur les conséquences de l'action protestataire sont en réalité passionnants, qu'on les pose au niveau des effets macro sociaux lourds (par exemple, les changements culturels), des orientations de politique publique ou encore des conséquences biographiques de l'engagement. Pour une revue de littérature, voir Didier Chabanet, Marco Giugni, «Les conséquences des mouvement sociaux », dans O. Fillieule, É. Agrikoliansky, I. Sommier (dir.), Penser les mouvements sociaux..., op. cit., p. 145-162.

${ }^{163} \mathrm{Ce}$ que font aussi les auteurs d'un ouvrage à paraître qui, comme ce dossier, rassemble des textes issus d'observations de première main et in situ: Amin Allal, Thomas Pierret (dir.), Devenir révolutionnaires. Au coeur des révoltes arabes, Paris, Armand Colin, 2013 à paraître.

${ }^{164}$ Carl F. Craver, « Mechanisms and Natural Kinds », Philosophical Psychology, 22 (5), 2009, p. 575-594.

165 M. Parsa, States, Ideologies..., op. cit.

${ }^{166}$ Anthony Oberschall, Social Conflict and Social Movements, Englewood Cliffs, Prentice Hall, 1973.
} 
pas facilement à des processus cognitifs, comme par exemple le courtage ${ }^{167}$.

Tableau. Quelques modes de transformation d'états propres aux situations révolutionnaires

\begin{tabular}{|c|c|c|c|}
\hline $\begin{array}{l}\text { Éléments conductifs } \\
\text { (présents en dehors du cadre temporel de la crise) }\end{array}$ & $\begin{array}{l}\text { Processus } \\
\text { (enchaînement d'échanges de coups entre } \\
\text { acteurs individuels et collectifs) }\end{array}$ & $\begin{array}{l}\text { Situation révolutionnaire } \\
\text { (situation de double pouvoir) }\end{array}$ & Échelle d'analyse \\
\hline $\begin{array}{l}\text { Structurels } \\
\text { Forme de l'État (+/-interventionniste, exclusif) } \\
\text { Forme du conflit de classe (+/-polarisé) } \\
\text { Segmentation verticale/horizontale } \\
\text { Mode de soutien international au régime } \\
\text { Dédifférenciation } \\
\text { Conjoncturels } \\
\text { Changement dans le mode de soutien au régime } \\
\text { Défaite militaire } \\
\text { Coup d'État, révolution de palais } \\
\text { Situation révolutionnaire ailleurs, etc. }\end{array}$ & & $\begin{array}{l}\text { Extension de la contestation à } \\
\text { une fraction significative de la } \\
\text { population } \\
\text { Brouillage des frontières entre } \\
\text { espaces sociaux } \\
\text { Vastes coalitions transclassistes } \\
\text { modularité des modes d'action et } \\
\text { des cadrages }\end{array}$ & $\begin{array}{c}\text { Macro } \\
\text { (environnementale) }\end{array}$ \\
\hline $\begin{array}{l}\text { Niveau de ressources des acteurs collectifs } \\
\text { (organisationnelles, leadership, idéologiques, etc.) } \\
\text { Capacité de courtage } \\
\text { Mémoire collective des luttes }\end{array}$ & $\begin{array}{l}\text { Attribution de similarité } \\
\text { Construction d'identités stratégiques } \\
\text { Cooptation } \\
\text { Concessions } \\
\text { Division } \\
\text { Répression ciblée/indiscriminée }\end{array}$ & $\begin{array}{l}\text { Division/défection dans les } \\
\text { organes centraux de } \\
\text { l'État/appareil répressif } \\
\text { Simplification des cadrages et } \\
\text { unification des modes d'action }\end{array}$ & $\begin{array}{c}\text { Méso } \\
\text { (relationnelle) }\end{array}$ \\
\hline $\begin{array}{l}\text { Mémoire individuelle des luttes } \\
\text { Socialisation/politisation } \\
\text { Position sociale } \\
\text { Confusion et incertitude croissante dans les anticipations }\end{array}$ & $\begin{array}{l}\text { Modification de l'équilibre préférences } \\
\text { publiques/privées } \\
\text { Émulation (évaluation des chances de succès) } \\
\text { Jeux d'assurance/dé-assurance } \\
\text { Effet et contre-effet de bandwagon } \\
\text { Critical mass/free rider }\end{array}$ & Évasion des calculs & $\begin{array}{c}\text { Micro } \\
\text { (cognitive) }\end{array}$ \\
\hline T0 & 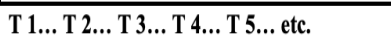 & $\mathrm{T} x$ & \\
\hline
\end{tabular}

La liste d'éléments dressée ici n'est qu'indicative et provisoire et devrait être complétée. Par ailleurs, il faudrait préciser mieux la distinction entre événements et processus. Si les processus se définissent comme des enchaînements d'échanges de coups entre acteurs individuels et collectifs, c'est donc qu'ils se composent aussi d'événements mais ne s'y réduisent pas. Dans l'état actuel, le tableau présenté ici devrait spécifier ce que nous entendons par répression comme processus (soit une stratégie déployée dans telle ou telle direction et sur la base de l'anticipation des coups des adversaires comme des alliés) et les événements répressifs comme coups joués à un moment donné du temps.

Il est clair enfin que les processus s'actualisent de manière variée en fonction des conditions de départ et qu'ils ne sont sans doute pas tous aussi centraux dans la survenance d'une situation révolutionnaire. Mais surtout, ils acquièrent leur force en se combinant les uns les autres, synchroniquement et diachroniquement. Si bien qu'un processus, à lui seul, n'explique rien. On le sait, les auteurs de Dynamics of contention ont essuyé la critique de naviguer de manière inconsistante entre la production de récits historiques et l'érection de certains mécanismes en variables explicatives ${ }^{168}$. Selon nous, c'est l'étude des séquences d'action et donc des combinaisons de processus activés et vécus par l'ensemble des parties prenantes qui permet de déplier les étapes successives qui, ici ou là, ont éventuellement conduit à des situations révolutionnaires. Ce faisant, l'on se tient à égale distance de

167 Tulia G. Falleti, Julia Lynch, «From Process to Mechanism. Varieties of Disaggregation », Qualitative sociology, 31 (3), 2008, p. 333-339.

168 Pamela Oliver décrit bien cette tension lorsqu'elle remarque que « a generalizing strategy focused on one process can never explain a historical episode, and should not try to. But conversely, a research program focused on explaining particular historical events cannot yield a general understanding of any process » («Mechanisms of Contention », Mobilization, 8 (1), 2003, p. 119-122, dont p. 121). Voir aussi Ruud Koopmans, «A Failed Revolution - but a Worthy Cause », Mobilization, 8 (1), 2003, p. 116-119, dont p. 117. 
l'établissement de lois du développement historique des situations révolutionnaires et du récit circonstancié et singulier de telle ou telle trajectoire révolutionnaire.

À titre d'exemple, des processus similaires sont à l'œuvre, au début de l'année 2011, en Égypte et au Maroc. Pour autant, ils ne se combinent pas à l'identique, conduisant dans le premier cas à une situation révolutionnaire, dans le second à une voie « réformiste ». Dans les deux pays s'observent des processus d'attribution de similarité, de construction d'identités stratégiques, se traduisant par la formation de coalitions par-delà les clivages et les polarisations préexistants. En revanche, les modalités de gestion de la crise s'agrègent différemment. Dans le cas égyptien, les concessions interviennent trop tardivement suite à une répression indiscriminée. Au Maroc, la proposition rapide d'une offre de réforme, le renforcement des capacités redistributives - entre autres grâce au soutien international -, le recours à une répression sélective et ponctuelle se combinent à la mise en place par les protestataires de dispositifs organisationnels permettant de «tenir la rue » et d'éviter les « débordements » qui ont conduit en Égypte au dépassement des initiateurs par les suiveurs. Autrement dit, au Maroc, l'autolimitation de l'ensemble des acteurs en présence contribue à inscrire la mobilisation dans la durée ; à l'inverse de l'Égypte, le point de non-retour n'est pas atteint et une voie du changement graduelle demeure à l'ordre du jour.

Reste pour terminer à revenir brièvement sur la manière la plus adéquate d'étudier les séquences d'action. Selon nous il est nécessaire d'articuler systématiquement description phénoménale et exploration du sens que les acteurs donnent à ce qu'ils font quand ils le font. C'est à ce prix et à ce prix seulement que le chercheur peut se garder de l'imputation de motivations aux individus à partir d'une observation de leurs seuls actes. R. Aya le fait sarcastiquement remarquer, sans cette attention au sens que les acteurs donnent à leurs actions aux différentes étapes d'un processus, l'analyse des situations ne procède en réalité pas des faits mais «d'hypothèses gratuites, de clichés sociologiques $»^{169}$. Dès lors la question se pose de savoir comment enregistrer des indices pour l'explication, c'est-à-dire « des preuves circonstanciées d'espoir de succès $\gg^{170}$. Les auteurs de ce dossier y apportent nous semble-t-il quelques réponses et nous indiquent au moins deux pistes.

En premier lieu, pour dépasser les imputations sauvages de sens et les classifications homogénéisantes autant que les supputations hors sol des approches pragmatiques, il faut s'attacher à une sociographie fine des acteurs dans leur diversité en replaçant leurs actes et leurs perceptions dans la perspective d'une histoire à la fois personnelle et familiale. Ce qui implique de placer au cour de l'analyse les acquis des travaux sur la socialisation et d'associer l'interrogation sur la formation des dispositions à agir à celle de leur transformation par l'expérience militante et l'événement ${ }^{17}$. Autrement dit, dans une perspective ni «causale » ni «linéaire », il conviendrait de mesurer ce que «l'engagement génère ou modifie des dispositions à agir, penser, percevoir - et se percevoir - en continuité ou en rupture avec les produits de socialisation antérieurs ${ }^{172}$. Pour ce faire, des enquêtes longitudinales s'imposent. Il faudra bien entendu attendre plusieurs années avant de pouvoir répondre à ces questions ${ }^{173}$, mais si l'on veut les aborder au moyen d'enquêtes longitudinales plutôt que rétrospectives, c'est aujourd'hui qu'il faut s'y atteler.

En second lieu, en appeler à une sociologie des situations révolutionnaires nécessite de se doter d'instruments d'observation et de méthodes qui permettent d'étudier les séquences

\footnotetext{
${ }^{169}$ R. Aya, «Agency... » [ou plutôt « The Third Man... » ?], art. cité. J. Bohstedt adresse cette même critique à E. P. Thompson à propos de la notion «d'économie morale » qu'il qualifie de romantique, alors qu'une attention pragmatique plus serrée aux performances émeutières lui aurait permis de mieux comprendre la place de l'invocation de la morale et la retenue des comportements dans les calculs des acteurs, émeutiers mais aussi représentants de l'ordre (John Bohstedt, «The Moral Economy and the Discipline of Historical Context », Journal of Social History, 26 (2), hiver 1992, p. 265-284).

${ }^{170}$ R. Aya, «Agency... » [ou plutôt « The Third Man... » ?], art. cité, p. 151.

${ }^{171}$ Olivier Fillieule, « Socialization and Social Movements », dans D. A. Snow, D. Della Porta, B. Klandermans, D. McAdam (eds), The Wiley-Blackwell Encyclopedia of Social and Political Movements Oxford, op. cit., à paraître.

${ }^{172}$ Catherine Leclercq, Julie Pagis, «Les incidences biographiques de l'engagement », Sociétés contemporaines, 84,2011 , p. 5-23, dont p. 5.

${ }^{173}$ D'après certains analystes, si les soulèvements arabes ont d'ores et déjà réalisé quelque chose, c'est en transformant profondément l'économie du consentement sur laquelle reposait la domination politique dans ces pays (L. Noueihed, A. Warren, The Battle for the Arab Spring..., op. cit., p. 134).
} 
d'action dans leur épaisseur phénoménale. De ce point de vue les matériaux visuels, accessibles sur Internet ${ }^{174}$, produits par le chercheur ou recueillis in situ sont précieux ${ }^{175}$, tout particulièrement dans des contextes où la production artistique (caricatures, graffitis, photos, vidéos) a joué un rôle non négligeable dans la dynamique protestataire ${ }^{176}$. Mais surtout, comme cela est souligné de plus en plus fréquemment ${ }^{177}$, les méthodes ethnographiques sont sans aucun doute les mieux à même de restituer la complexité des échanges de coup et des calculs à chaque étape des séquences d'action. Or la littérature en sociologie des révolutions, et plus généralement en sociologie des mobilisations, est là particulièrement pauvre. De ce point de vue, les pistes ouvertes par des auteurs aussi divers que D. Snow et L. Anderson, B. Roy, A. Peterson et bien sûr P. Lichterman et J. Auyero ${ }^{178}$ n'ont pas encore à ce jour suscité un tournant ethnographique en sociologie des mouvements sociaux et des révolutions, malgré de rares mais notables exceptions ${ }^{179}$.

Enfin, et nous espérons que l'esprit comme la forme du dossier qu'on va lire le manifeste assez, face à la densité des sources, décortiquer les séquences d'action conduisant aux situations révolutionnaires nécessite à l'évidence de renoncer à la recherche en solitaire et de constituer des équipes pluridisciplinaires, dotées de compétences linguistiques et informatiques ${ }^{180}$, mais aussi d'une connaissance approfondie de l'historicité des espaces

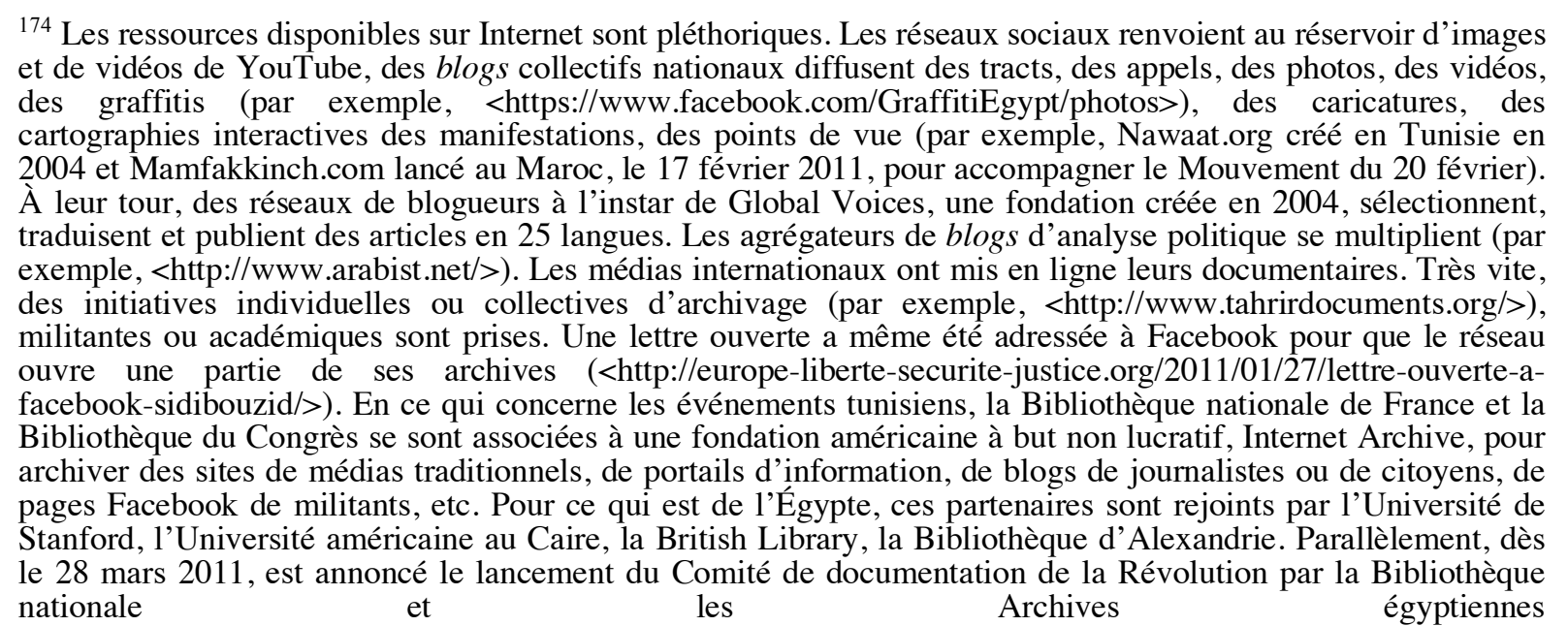
$\begin{array}{lll}\text { nationale et } & \text { Archives } & \text { égyptiennes } \\ \text { (<http://www.aucegypt.edu/newsatauc/Pages/story.aspx?eid=640;http://blog.bnf.fr/lecteurs/index.php/2012/07/0 }\end{array}$ 2/la-revolution-du-jasmin-sur-la-toile/;http://www.archive-it.org/public/collection.html?id=2323>).

175 Alexandre Lambelet, «Analyser les rassemblements au moyen de photographies ou de films. Pistes et enjeux », ethnographiques.org, 21, 2010, <http://www.ethnographiques.org/2010/Lambelet> ; Alex Philipps, «Visual Protest Material as Empirical Data », Visual Communication, 11 (3), 2012, p. 3-21.

${ }^{176}$ Voir entre autres Anahi Alviso-Marino, «Art et révolution : engagement des artistes dans la mobilisation contestataire au Yémen », à paraître ; Cécile Boëx, «L'engagement des acteurs, des cinéastes et des comédiens dans la révolte en Syrie », dans A. Allal, T. Pierret (dir.), Devenir révolutionnaires..., op. cit., p. ?- ?.

177 Par exemple, Suzanne Staggenborg, « Seeing Mechanisms in Action », Qualitative Sociology, 31 (4), 2008, p. 341-344 ; O. Fillieule, É. Agrikoliansky, I. Sommier (dir.), Penser les mouvements sociaux..., op. cit.; Hélène Combes et al., « Observer les mobilisations », Politix, 93, 2011, p. 7-27.

178 David A. Snow, Leon Anderson, Down on Their Luck. A Study of Homeless Street People, Berkeley, University of California Press, 1993, sur les mobilisations autour des sans domicile fixe à Austin; Paul Lichterman, The Search for Political Community. American Activists Reinventing Commitment, New York, Cambridge University Press, 1996, sur l'activisme communautaire en Californie ; Anna L. Peterson, Martyrdom and the Politics of Religion. Progressive Catholicism in El Salvador's Civil War, Albany, SUNY Press, 1997, sur les mouvements catholiques progressistes au Salvador; Javier Auyero, Contentious Lives. Two Argentine Women, Two Protests, and the Quest for Recognition, Durham, Duke University Press, 2003, sur les parcours de politisation de deux femmes argentines.

${ }^{179}$ Notamment Elisabeth Jean Wood, Insurgent Collective Action and Civil War in El Salvador, New York, Cambridge University Press, 2003; Ann Mische, Partisan Publics. Communication and Contention Across Brazilian Youth Activist Networks, Princeton, Princeton University Press, 2008.

${ }_{180}$ Par exemple, si les sociologues des mobilisations et des révolutions aspirent à étendre leur terrain à l'univers du virtuel, ils devront se confronter à de nouveaux problèmes techniques et méthodologiques tant sur le plan de l'accès aux sources et de leur récolte qu'à celui des modes de traitements des «big data ». Des réflexions dans ce sens sont menées dans le cadre d'un groupe de chercheurs en «Humanités digitales » de Lausanne (<http://www3.unil.ch/wpmu/digitalera/>). 
étudiés. De ce point de vue, l'idée défendue ici de centrer l'observation sur les situations révolutionnaires ne doit pas être comprise comme une invitation à faire l'économie d'une sociohistoire des pratiques et d'une sociologie des carrières militantes.

Sollicité pour donner son avis sur la Révolution française de 1789, Zhou Enlai aurait malicieusement répondu qu'il était trop tôt pour en juger. Conclure l'introduction du présent dossier sur les soulèvements arabes en cours par cette vignette apocryphe pleine de sagesse dit bien à quel point nous n'en sommes qu'aux balbutiements d'une telle entreprise ${ }^{181}$.

Mounia Bennani-Chraïbi est professeure associée en politique comparée et en sociologie des mobilisations en Afrique du Nord et au Moyen-Orient à l'Institut d'études politiques et internationales de l'Université de Lausanne, et membre du CRAPUL (Centre de recherche sur l'action politique de l'Université de Lausanne). La liste de ses activités de recherche et de ses publications est disponibles sur le site de l'UNIL: <http://www.unil.ch/unisciences/MouniaBennani-Chraibi> (Université de Lausanne, IEPI, Géopolis, 1015 Lausanne, Suisse <Mounia.Bennani@unil.ch>).

Directeur de recherche CNRS, Olivier Fillieule est professeur ordinaire de sociologie politique à l'Institut d'études politiques et internationales de l'Université de Lausanne et membre du CRAPUL (Centre de recherche sur l'action politique de l'Université de Lausanne). La liste de ses activités de recherche et de ses publications est disponible sur son site personnel : <http://people.unil.ch/olivierfillieule/> (Université de Lausanne, IEPI, Géopolis, 1015 Lausanne, Suisse <olivier.fillieule@unil.ch>).

${ }^{181}$ Les auteurs tiennent à remercier pour leur lecture stimulante Philip Balsiger, Dina El-Khawaga, Pierre Favre, Choukri Hmed et les évaluateurs de la revue. L'ordre des auteurs est alphabétique. 